TRANSACTIONS OF THE

AMERICAN MATHEMATICAL SOCIETY

Volume 357, Number 4, Pages 1275-1301

S 0002-9947(04)03718-3

Article electronically published on November 23, 2004

\title{
SUBSMOOTH SETS: FUNCTIONAL CHARACTERIZATIONS AND RELATED CONCEPTS
}

\author{
D. AUSSEL, A. DANIILIDIS, AND L. THIBAULT
}

\begin{abstract}
Prox-regularity of a set (Poliquin-Rockafellar-Thibault, 2000), or its global version, proximal smoothness (Clarke-Stern-Wolenski, 1995) plays an important role in variational analysis, not only because it is associated with some fundamental properties as the local continuous differentiability of the function $\operatorname{dist}(C ; \cdot)$, or the local uniqueness of the projection mapping, but also because in the case where $C$ is the epigraph of a locally Lipschitz function, it is equivalent to the weak convexity (lower- $\mathrm{C}^{2}$ property) of the function. In this paper we provide an adapted geometrical concept, called subsmoothness, which permits an epigraphic characterization of the approximate convex functions (or lower- $\mathrm{C}^{1}$ property). Subsmooth sets turn out to be naturally situated between the classes of prox-regular and of nearly radial sets. This latter class has been recently introduced by Lewis in 2002 . We hereby relate it to the Mifflin semismooth functions.
\end{abstract}

\section{INTRODUCTION}

Let $X$ be a real Banach space and let $U$ be a non-empty open subset of $X$. A function $f: U \rightarrow \mathbb{R}$ is called weakly convex on $U$ if for every $x_{0} \in U$ there exists $\sigma, \delta>0$ such that, for every $x, y \in B\left(x_{0}, \delta\right)$ and $t \in[0,1]$,

$$
f(t x+(1-t) y) \leq t f(x)+(1-t) f(y)+\sigma t(1-t)\|x-y\|^{2} .
$$

This class of functions, initially introduced by Vial [33], has been extensively used by many authors under various equivalent definitions (see [15] and references therein) and enjoys various interesting properties. For example, every continuous function satisfying (1.1) can be decomposed to a difference of a convex continuous function and a convex quadratic function ([33, Proposition 4.3]). Another important equivalent property, from the application point of view, is the following one, established by Rockafellar 28 in finite dimensions: a (locally Lipschitz) function $f$ is weakly convex if, and only if, it is lower- $\mathrm{C}^{2}$. We recall that a function $f$ is called lower- $\mathrm{C}^{k}$ on $U(k \in \mathbb{N})$ if for every $x_{0} \in U$ there exist a neighbourhood $V$ of $x_{0}$, a compact topological space $S$, and a jointly continuous function $g: V \times S \rightarrow \mathbb{R}$ satisfying

$$
f(x)=\max _{s \in S} g(x, s) \quad \text { for all } x \in V,
$$

Received by the editors February 24, 2003.

2000 Mathematics Subject Classification. Primary 26B25; Secondary 49J52, 47H04.

Key words and phrases. Variational analysis, subsmooth sets, submonotone operator, approximately convex functions.

The research of the second author has been supported by the Spanish Ministry of Education Program: "Ayudas para estancias de profesores e investigadores extranjeros en España" (Grant No SB2000-0369). 
and such that all derivatives of $g$ of order $k$ with respect to $x$ (exist and) are jointly continuous ([28] e.g.). This class of functions provides a robust and stable extension of both the notion of convex function and the notion of smooth function. Their role in optimization is important; see [15] for a survey. For lower- $\mathrm{C}^{k}$ functions in Hilbert space, we refer to [24].

In the same period, several extensions of convexity (respectively, of monotonicity) have been proposed for sets; see [33], 30] and references therein (respectively, for multivalued operators, see 28], [31]). In this line of research, Clarke, Stern and Wolenski studied in [5] the notion of proximal smoothness. They proved that a closed set $C$ of a Hilbert space is proximally smooth (or uniformly prox-regular, according to [25]) if, and only if, its square distance function $\operatorname{dist}(., C)^{2}$ is continuously differentiable in an open tube of uniform thickness around $C$ (we also refer to Federer in 14 for the finite-dimensional case, where such sets are called positively reached). They have also shown that this property characterizes the epigraphs of lower- $\mathrm{C}^{2}$ functions (in finite dimensions), establishing an interesting link between sets and functions (see also [33, Propositions 4.17, 4.18]). Poliquin, Rockafellar and Thibault in [25] developing the corresponding local theory under the name of prox-regularity, established another interesting characterization based on the hypomonotonicity of the truncated Clarke normal cone $N^{r}(C ;$.$) of C$, where $r>0$. The same property, that is, hypomonotonicity, had been previously used to characterize the subdifferentials of weakly convex functions (28). Thus, in finite dimensions, one has the following complete geometrical characterization of locally Lipschitz weakly convex functions (this characterization will be extended in Hilbert spaces in Section 4):

$$
f \text { is weakly convex } \Leftrightarrow \partial f \text { is hypomonotone } \Leftrightarrow \text { epi } f \text { is prox-regular. }
$$

The weaker notion of approximate convexity has been recently considered by Ngai, Luc and Théra [23]. We also refer to Rolewicz [29] and references therein for some similar convex-like functions. According to the definition in [23, a function $f: U \rightarrow \mathbb{R}$ is called approximately convex on $U$ if for every $x_{0} \in U$ and every $\varepsilon>0$, there exists $\delta>0$ such that for every $x, y \in B\left(x_{0}, \delta\right)$ and for every $t \in[0,1]$

$$
f(t x+(1-t) y) \leq t f(x)+(1-t) f(y)+\varepsilon t(1-t)\|x-y\| .
$$

This class of functions satisfies interesting integration properties, is stable under finite sums and finite suprema and enjoys exact subdifferential sum rules (see [23]). In 9], Daniilidis and Georgiev emphasized that, in finite dimensions, this class of functions coincide with the well-known class of lower- $\mathrm{C}^{1}$ functions (see [31] or [28] for an extensive study of this latter class). Moreover, (locally Lipschitz) approximately convex functions have been characterized by the submonotonicity of their subdifferentials.

By introducing the geometrical concept of subsmoothness of the set related to the submonotonicity of the truncated Clarke normal cone, our aim is to establish the statements analogous to (1.2) for approximately convex functions, namely:

$f$ is approximately convex $\Leftrightarrow \partial f$ is submonotone $\Leftrightarrow$ epi $f$ is subsmooth.

Section 4 is essentially devoted to this task (see Theorem 4.14 and Corollary 4.17). The notion of a subsmooth set is introduced in section 3 for an arbitrary closed set (Definition 3.1). Here we shed light on the links with other geometrical notions, in particular with the tangential Shapiro property and the notion of "near 
radiality", introduced recently by Lewis [18] (see Theorem [3.16). We relate this latter notion to Mifflin semismooth functions, providing an interesting link to functions satisfying a property of type (1.3) (see Theorem [3.16(c), Theorem 4.9 and Theorem 4.15). In section 2, we give some preliminaries and we state some useful properties of different kinds of normal cones of variational analysis.

\section{Preliminaries}

Throughout the manuscript $X$ denotes a Banach space, $X^{*}$ its topological dual and $B\left(x_{0}, \delta\right)$ (respectively, $B\left[x_{0}, \delta\right]$ ) the open (respectively, closed) ball of center $x_{0} \in X$ and radius $\delta>0$. If $C$ is a nonempty subset of $X$, the (Clarke) normal cone of $C$ at $u \in C$ is defined by

$$
N(C ; u)=\left\{x^{*} \in X^{*}:\left\langle x^{*}, v\right\rangle \leq 0, \forall v \in T(C ; u)\right\},
$$

where the Clarke tangent cone $T(C ; u)$ is defined as follows:

$$
v \in T(C ; u) \Longleftrightarrow\left\{\begin{array}{l}
\forall \varepsilon>0, \exists \delta>0 \text { such that } \\
\left.\forall u^{\prime} \in B(u, \delta) \cap C, \forall t \in\right] 0, \delta\left[,\left(u^{\prime}+t B(v, \varepsilon)\right) \cap C \neq \emptyset .\right.
\end{array}\right.
$$

We put $N(C ; u)=\emptyset$, whenever $u \notin C$. For any $r>0$ we denote by $N^{r}(C ; u)$ the truncated Clarke normal cone, that is,

$$
N^{r}(C ; u)=N(C ; u) \cap B[0, r] .
$$

The set $C$ is called tangentially regular at $u \in C$ if $T(C ; u)=K(C ; u)$, where $K(C ; u)$ stands for the Bouligand tangent cone to $C$ at $u$, that is,

$$
v \in K(C ; u) \Longleftrightarrow \forall \delta>0, \exists t \in(0, \delta) \text { such that }(u+t B(v, \delta)) \cap C \neq \emptyset .
$$

The concept of Fréchet normal vectors will be also needed. A vector $u_{0}^{*}$ is Fréchet normal to $C$ at $u_{0} \in C$ if for every $\varepsilon>0$ there exists $\delta>0$ such that for all $u \in C$ with $\left\|u-u_{0}\right\|<\delta$ one has $\left\langle u_{0}^{*}, u-u_{0}\right\rangle \leq \varepsilon\left\|u-u_{0}\right\|$. The cone of all Fréchet normal vectors to $C$ at $u_{0}$ will be denoted by $N_{F}\left(C ; u_{0}\right)$. We will also put $N_{F}(C ; u)=\emptyset$ when $u \notin C$. We recall that $C$ is normally Fréchet regular at $u_{0} \in C$ whenever the Clarke and the Fréchet normal cone to $C$ at $u_{0}$ coincide. If the set $C$ is normally Fréchet regular at $u_{0}$, then it is also tangentially regular ([3, Theorem 6.2]).

We typically denote by $f: X \rightarrow \mathbb{R} \cup\{+\infty\}$ a proper function (that is, $f$ is finite at least at one point) with domain $\operatorname{dom} f:=\{x \in X: f(x) \in \mathbb{R}\}$ and epigraph epif $:=\{(x, t) \in X \times \mathbb{R}: f(x) \leq t\}$. The Clarke subdifferential $\partial f\left(x_{0}\right)$ of a locally Lipschitz function $f$ at $x_{0}$ is defined as follows:

$$
\partial f\left(x_{0}\right)=\left\{x^{*} \in X:\left(x^{*}, v\right) \leq f^{0}\left(x_{0} ; v\right), \forall v \in X\right\},
$$

where

$$
f^{0}\left(x_{0} ; v\right):=\limsup _{(y, t) \rightarrow\left(x_{0}, 0^{+}\right)} \frac{f(y+t v)-f(y)}{t} .
$$

A locally Lipschitz function is called directionally regular (in short, d-regular) at $x_{0}$, if for all $v \in X$,

$$
f^{0}\left(x_{0} ; v\right)=d^{-} f\left(x_{0} ; v\right), \quad \text { where } d^{-} f\left(x_{0} ; v\right)=\liminf _{t \searrow 0^{+}} \frac{f\left(x_{0}+t v\right)-f\left(x_{0}\right)}{t} .
$$

A vector $x^{*} \in X^{*}$ is said to be in the Fréchet subdifferential $\partial_{F} f\left(x_{0}\right)$ of $f$ at $x_{0}$ provided that for every $\varepsilon>0$ there exists $\delta>0$ such that for all $x \in B\left(x_{0}, \delta\right)$ one has

$$
\left\langle x^{*}, x-x_{0}\right\rangle \leq f(x)-f\left(x_{0}\right)+\varepsilon\left\|x-x_{0}\right\| .
$$


If $\psi_{C}$ denotes the indicator function of the set $C$, that is, $\psi_{C}(x)=0$ if $x \in C$ and $\psi_{C}(x)=+\infty$ otherwise, then it is easily seen that

$$
N_{F}(C ; u)=\partial_{F} \psi_{C}(u) .
$$

The main properties of Fréchet normal cones and Fréchet subdifferentials require that the Banach space is Asplund. We recall that a Banach space $X$ is called Asplund (11 e.g.) if every separable subspace of $X$ has a separable topological dual. In particular, every reflexive Banach space is Asplund. In Asplund spaces, the limiting (Mordukhovich) subdifferential $\partial_{L} f$ is defined as follows:

$$
\partial_{L} f\left(x_{0}\right)=\mathrm{w}^{*} \text {-seq- } \limsup _{x \rightarrow x_{0}} \partial_{F} f(x),
$$

where $\mathrm{w}^{*}$-seq-lim sup denotes the weak star sequential outer (superior) limit. This $x \rightarrow f x_{0}$

means that $x^{*} \in \partial_{L} f\left(x_{0}\right)$ whenever there are sequences $x_{n} \rightarrow_{f} x$ and $x_{n}^{*} \in \partial_{F} f\left(x_{n}\right)$ with $\left(x_{n}^{*}\right)$ converging to $x^{*}$ with respect to the weak star topology of $X^{\star}$. (By $x_{n} \rightarrow_{f} x$ we mean that $x_{n} \rightarrow x$ and $f\left(x_{n}\right) \rightarrow f(x)$.)

Similarly, one defines the limiting normal cone $N_{L}\left(C ; u_{0}\right)$ as follows:

$$
N_{L}\left(C ; u_{0}\right)=\text { w }^{*} \text {-seq- } \limsup _{u \rightarrow C u_{0}} N_{F}(C ; u) .
$$

(By $u \rightarrow_{C} u_{0}$ we mean that the above limit is taken in $C$.) As in the previous cases, $N_{L}(C ; u)=\emptyset$ when $u \notin C$. For the indicator function $\psi_{C}$ of $C$, it is easily seen that

$$
N_{L}\left(C ; u_{0}\right)=\partial_{L} \psi_{C}\left(u_{0}\right) .
$$

The distance of a point $x \in X$ from $C$ is defined by

$$
\operatorname{dist}(C ; x)=\inf \{\|x-y\|: y \in C\} .
$$

The link between the Fréchet normal cone and the distance function is given by the following equality of [16] (see also [3] and references therein):

$$
N_{F}^{1}\left(C ; u_{0}\right)=\partial_{F} \operatorname{dist}(C ; .)\left(u_{0}\right), \quad \text { for } u_{0} \in C,
$$

where, as for the Clarke normal cone,

$$
N_{F}^{r}\left(C ; u_{0}\right)=N_{F}\left(C ; u_{0}\right) \cap B[0, r], \quad \text { for any } r>0 .
$$

Concerning the Clarke normal cone, one has the equality (see 4])

$$
N\left(C ; u_{0}\right)=w^{*}-\operatorname{cl}\left(\mathbb{R}_{+} \partial \operatorname{dist}(C ; .)\left(u_{0}\right)\right),
$$

where $w^{*}$-cl denotes the topological closure with respect to the weak star topology of $X^{*}$.

In Asplund spaces the following equalities also hold ([21]):

$$
N\left(C ; u_{0}\right)=w^{*}-\operatorname{clco} N_{L}\left(C ; u_{0}\right)
$$

and

$$
\partial \operatorname{dist}(C ; .)\left(u_{0}\right)=w^{*} \text {-cl co } \partial_{L} \operatorname{dist}(C ; .)\left(u_{0}\right),
$$

where $w^{*}$-cl co denotes the weak star closed convex hull. 


\section{Subsmooth Sets And Related GeOMEtrical CONCEPTS}

3.1. Definitions and elementary properties. Let $C$ be a nonempty closed subset of a Hilbert space and let $u_{0} \in C$. We recall from [25] that the set $C$ is called prox-regular at $u_{0}$, if for every $r>0$, there exists $\sigma, \delta>0$ such that for all $u_{1}, u_{2} \in B\left(u_{0}, \delta\right) \cap C$ and all $u_{i}^{*} \in N^{r}\left(C ; u_{i}\right), i \in\{1,2\}$, one has

$$
\left\langle u_{1}^{*}-u_{2}^{*}, u_{1}-u_{2}\right\rangle \geq-\sigma\left\|u_{1}-u_{2}\right\|^{2}
$$

This turns out to be equivalent to saying that the distance function $\operatorname{dist}(C,$.$) is$ continuously differentiable on $U \backslash C$, for some open neighbourhood $U$ of $u_{0}$. Further, $C$ is called prox-regular if it is prox-regular at every $u_{0} \in C$ and uniformly prox-regular if there exists $\sigma>0$ such that (3.1) holds for all $u_{1}, u_{2} \in C$. According to [25, Theorem 4.1], $C$ is uniformly prox-regular if, and only if, the square distance function $\operatorname{dist}(C, .)^{2}$ is continuously differentiable on an open tube of uniform thickness around $C$. So, uniformly prox-regular sets correspond to what is called proximally smooth sets in [5].

We now introduce a new class of sets via the concept of subsmoothness. It can be seen as a variational behavior of "order one" of the set $C$, while 3.1 is expressing a variational behavior of "order two". In this paper, $C$ will be a closed subset of the Banach space $X$.

Definition 3.1. The set $C$ is called subsmooth at $u_{0}$ if for every $r>0$ and every $\varepsilon>0$ there exists $\delta>0$ such that for all $u_{1}, u_{2} \in B\left(u_{0}, \delta\right) \cap C$ and all $u_{i}^{*} \in N^{r}\left(C ; u_{i}\right)$, $i \in\{1,2\}$, one has

$$
\left\langle u_{1}^{*}-u_{2}^{*}, u_{1}-u_{2}\right\rangle \geq-\varepsilon\left\|u_{1}-u_{2}\right\| .
$$

The set $C$ is called subsmooth if it is subsmooth at every $u_{0} \in C$.

We further say that $C$ is uniformly subsmooth if for every $r>0$ and $\varepsilon>0$ there exists $\delta>0$, such that (3.2) holds for all $u_{1}, u_{2} \in C$ satisfying $\left\|u_{1}-u_{2}\right\|<\delta$ and all $u_{i}^{*} \in N^{r}\left(C ; u_{i}\right), i \in\{1,2\}$.

By imposing $u_{2}=u_{0}$ in (3.2) one results with a radial counterpart of the above concept that we call semi-subsmoothness. Let us state the exact definition.

Definition 3.2. The set $C$ is called semi-subsmooth at $u_{0}$ if for every $r>0$ and $\varepsilon>0$ there exists $\delta>0$ such that for all $u \in B\left(u_{0}, \delta\right) \cap C, u_{0}^{*} \in N^{r}\left(C ; u_{0}\right)$ and $u^{*} \in N^{r}(C ; u)$, we have

$$
\left\langle u^{*}-u_{0}^{*}, u-u_{0}\right\rangle \geq-\varepsilon\left\|u-u_{0}\right\| .
$$

The set $C$ is called semi-subsmooth if it is semi-subsmooth at every $u_{0} \in C$.

Note that the natural definition for a notion of uniform semi-subsmoothness will yield the previously defined notion of uniform subsmoothness.

Remark 3.3. (i) Conditions (3.1), 3.2) and (3.3) are equivalent to the statements arising by taking $r=1$ (respectively, $\varepsilon=1$ ).

(ii) Every set $C$ is prox-regular (respectively, subsmooth, semi-submonotone) at any point of $\operatorname{int} C$.

The following result makes the connection between subsmoothness and other classical geometrical concepts. 
Proposition 3.4. (i) Uniformly prox-regular sets are also uniformly subsmooth.

(ii) Every prox-regular set $C\left(\right.$ at $\left.u_{0}\right)$ is subsmooth (at $u_{0}$ ) and every subsmooth set (at $u_{0}$ ) is semi-subsmooth (at $\left.u_{0}\right)$.

(iii) If $C$ is semi-subsmooth at $u_{0}$, then it is normally Fréchet regular, and consequently tangentially regular, at $u_{0}$.

Proof. Statements $(i)$ and $(i i)$ are straightforward from the definitions. To establish (iii), let us assume that $C$ is semi-subsmooth at $u_{0} \in C$. Fix any $u_{0}^{*} \in N\left(C ; u_{0}\right)$ and let $r>\left\|u_{0}^{*}\right\|$. Also fix any $\varepsilon>0$. Choose $\delta>0$ such that (3.3) holds. Taking $u^{*}=0 \in N^{r}(C ; u)$, we obtain for all $u \in B\left(u_{0}, \delta\right) \cap C$

$$
\left\langle u_{0}^{*}, u-u_{0}\right\rangle \leq \varepsilon\left\|u-u_{0}\right\| .
$$

This translates into the fact that $u_{0}^{*}$ is a Fréchet normal to $C$ at $u_{0}$ and hence $N\left(C ; u_{0}\right)$ is included in the Fréchet normal cone $N_{F}\left(C ; u_{0}\right)$. In fact the equality holds because the reverse inclusion is always true. The tangential regularity result follows from [3, Theorem 6.2].

3.2. The limiting subdifferential of the distance function. In Asplund spaces the limiting subdifferential $\partial_{L} f$ of a locally Lipschitz function $f$ is provided by the Fréchet subdifferential $\partial_{F} f$ via the formula (2.6). The aim of this paragraph is to establish a refinement of the aforementioned formula, for the particular case that $f(x)=\operatorname{dist}(C ; x)$, where $C$ is a non-empty closed set. Namely, the limit in (2.6) is considered only in $C$ (instead of $X$ ). This important fact will be used in Section 3.3 to provide alternative characterizations of subsmooth sets and to facilitate comparison with other concepts.

Let us state this result.

Proposition 3.5. Let $C$ be a closed subset of an Asplund space $X$ and let $u_{0} \in C$. Then

$$
\partial_{L} \operatorname{dist}(C ; .)\left(u_{0}\right)=\mathrm{w}^{*} \text {-seq-limsup } \sup _{u \rightarrow C} \partial_{0} \operatorname{dist}(C ; .)(u) .
$$

Before giving the proof, we need to recall the following important fuzzy calculus result, due to Fabian (see [13]).

Proposition 3.6. Let $X$ be an Asplund space, let $g: X \rightarrow \mathbb{R}$ be a locally Lipschitz function, and let $f: X \rightarrow \mathbb{R} \cup\{+\infty\}$ be a lower semicontinuous function that is finite at $x_{0}$. Then for any $\eta>0$ there are $x, y \in B\left(x_{0}, \eta\right)$ with $\left|f(x)-f\left(x_{0}\right)\right|<\eta$ such that

$$
\partial_{F}(f+g)\left(x_{0}\right) \subset \partial_{F} f(x)+\partial_{F} g(y)+B[0, \eta] .
$$

The proof of Proposition 3.5 is based on the next lemma, which also has an independent interest. Its proof is inspired from [32] (see also [19] for some other properties and 22] for Banach spaces with smooth renorms).

Lemma 3.7. Let $C$ be a closed subset of an Asplund space $X$, let $x \in X$ and let $x^{*} \in$ $\partial_{F} \operatorname{dist}(C ;).(x)$. Then for every $\varepsilon>0$, there exist $u \in C$ and $u^{*} \in \partial_{F} \operatorname{dist}(C ;).(u)$ such that

$$
\|u-x\| \leq \varepsilon+\operatorname{dist}(C ; x) \quad \text { and } \quad\left\|u^{*}-x^{*}\right\| \leq \varepsilon .
$$

Proof. Fix $\varepsilon>0$ and choose $\eta>0$ with $\eta<\min (1, \varepsilon / 6)$. By definition of the Fréchet subdifferential (2.5), we may choose some positive number $\alpha<\eta / 2$ such that for all $x^{\prime} \in B[x, \alpha]$

$$
\left\langle x^{*}, x^{\prime}-x\right\rangle \leq \operatorname{dist}\left(C ; x^{\prime}\right)-\operatorname{dist}(C ; x)+\eta\left\|x^{\prime}-x\right\| .
$$


Fix some $y \in C$ satisfying

$$
\|x-y\| \leq \alpha^{2}+\operatorname{dist}(C ; x) .
$$

Then for all $y^{\prime} \in B[y, \alpha]$ we have by (3.4)

$$
\begin{aligned}
\left\langle x^{*}, y^{\prime}-y\right\rangle & \leq \operatorname{dist}\left(C ; x+y^{\prime}-y\right)-\operatorname{dist}(C ; x)+\eta\left\|y^{\prime}-y\right\| \\
& \leq \operatorname{dist}\left(C ; y^{\prime}\right)+\|x-y\|-\operatorname{dist}(C ; x)+\eta\left\|y^{\prime}-y\right\|
\end{aligned}
$$

and hence according to 3.5

$$
\left\langle x^{*}, y^{\prime}-y\right\rangle \leq \operatorname{dist}\left(C ; y^{\prime}\right)+\eta\left\|y^{\prime}-y\right\|+\alpha^{2} .
$$

Consequently, this yields for all $y^{\prime} \in C \cap B[y, \alpha]$

$$
\left\langle x^{*}, y^{\prime}-y\right\rangle \leq \eta\left\|y^{\prime}-y\right\|+\alpha^{2} .
$$

Set $\varphi(x)=\left\langle-x^{*}, x\right\rangle+\eta\|x-y\|$, so that $\varphi(y) \leq \varphi\left(y^{\prime}\right)+\alpha^{2}$, for all $y^{\prime} \in C \cap B[y, \alpha]$. Applying the Ekeland variational principle (see [12]) with $\lambda=\alpha / 2$ we obtain some $z \in C \cap B[y, \alpha]$ with $\|z-y\| \leq \lambda$ and such that for all $z^{\prime} \in C \cap B[y, \alpha]$

$$
\varphi(z) \leq \varphi\left(z^{\prime}\right)+\frac{\alpha^{2}}{\lambda}\left\|z^{\prime}-z\right\|
$$

that is,

$$
\left\langle-x^{*}, z\right\rangle+\eta\|z-y\| \leq\left\langle-x^{*}, z^{\prime}\right\rangle+\eta\left\|z^{\prime}-y\right\|+\frac{\alpha^{2}}{\lambda}\left\|z^{\prime}-z\right\|,
$$

and hence

$$
\left\langle x^{*}, z^{\prime}-z\right\rangle \leq \eta\left(\left\|z^{\prime}-y\right\|-\|z-y\|\right)+2 \alpha\left\|z^{\prime}-z\right\| \leq 2 \eta\left\|z^{\prime}-z\right\| .
$$

Set $\Phi(x)=\psi_{C}(x)+2 \eta\|x-z\|$ and observe that $B(z, \alpha / 2) \subset B[y, \alpha]$. Thus (3.6) yields that

$$
\left\langle x^{*}, z^{\prime}-z\right\rangle \leq \Phi\left(z^{\prime}\right)-\Phi(z), \quad \text { for all } z^{\prime} \in B(z, \alpha / 2) .
$$

This implies that

$$
x^{*} \in \partial_{F} \Phi(z)=\partial_{F}\left(\psi_{C}(.)+2 \eta\|.-z\|\right)(z) .
$$

According to Proposition [3.6. the latter entails the existence of some $u \in C$ with $\|u-z\|<\eta$ and such that $x^{*} \in \partial_{F} \psi_{C}(u)+3 \eta B[0,1]=N_{F}(C ; u)+3 \eta B[0,1]$. Then we may choose some $e^{*} \in B[0,1]$ satisfying

$$
v^{*}:=x^{*}+3 \eta e^{*} \in N_{F}(C ; u) .
$$

Since $x^{*} \in \partial_{F} \operatorname{dist}(C ;).(x)$, we have $\left\|x^{*}\right\| \leq 1$, thus $\left\|v^{*}\right\| \leq 1+3 \eta$. Applying (2.7) we get

$$
u^{*}:=(1+3 \eta)^{-1} v^{*} \in \partial_{F} \operatorname{dist}(C ; .)(u) .
$$

Further, since $\left\|x^{*}-e^{*}\right\| \leq 2$ we have

$$
\left\|u^{*}-x^{*}\right\|=\frac{3 \eta}{1+3 \eta}\left\|x^{*}-e^{*}\right\| \leq \frac{6 \eta}{1+3 \eta}<\varepsilon .
$$

On the other hand, by 3.5

$$
\|u-x\| \leq\|u-z\|+\|z-y\|+\|y-x\| \leq \eta+\alpha+\alpha^{2}+\operatorname{dist}(C ; x),
$$

which implies

$$
\|u-x\|<\varepsilon+\operatorname{dist}(C ; x) .
$$

The proof is complete. 
Proof of Proposition 3.5. According to the definition of the limiting subdifferential (see (2.6) ), the second member is included in the first one. For the converse inclusion, let $u_{0}^{*} \in \partial_{L} \operatorname{dist}(C ;).\left(u_{0}\right)$. Then there exist $\left\{x_{n}\right\}_{n \geq 1}$ in $X,\left\{x_{n}^{*}\right\}_{n \geq 1}$ in $X^{*}$, with $x_{n}^{*} \in \partial_{F} \operatorname{dist}(C ;).\left(x_{n}\right)$, such that

$$
\|\cdot\|-\lim _{n \rightarrow+\infty} x_{n}=u_{0} \quad \text { and } \quad w^{*}-\lim _{n \rightarrow+\infty} x_{n}^{*}=u_{0}^{*} .
$$

Applying Lemma [3.7] for every $n \geq 1$ and for $\varepsilon_{n}=1 / n$, we obtain a sequence $\left\{u_{n}\right\}_{n \geq 1}$ in $C$, and a sequence $\left\{u_{n}^{*}\right\}_{n \geq 1}$ in $X^{*}$ with $u_{n}^{*} \in \partial_{F} \operatorname{dist}(C ;).\left(u_{n}\right)$, such that for all $n \geq 1$

$$
\left\|u_{n}-x_{n}\right\| \leq \varepsilon_{n}+\operatorname{dist}\left(C ; x_{n}\right) \quad \text { and } \quad\left\|u_{n}^{*}-x_{n}^{*}\right\| \leq \varepsilon_{n} .
$$

It follows that $\|\cdot\|-\lim _{n \rightarrow+\infty} u_{n}=u_{0}$ and $w^{*}-\lim _{n \rightarrow+\infty} u_{n}^{*}=u_{0}^{*}$. The proof is complete.

3.3. Subsmoothness vs. Fréchet normals. The aim of this section is to provide alternative characterizations of subsmoothness in terms of Fréchet normals and of subdifferentials of the distance functions. These characterizations, stated for reflexive Banach spaces, are partially based on the results of the previous section. To start with, let $C$ be a closed subset of $X$ and let $u_{0} \in C$. The following lemma will be used in the sequel.

Lemma 3.8. (i) If $\partial \operatorname{dist}(C ;$.$) satisfies (3.2) at u_{0}$, then

$$
\partial \operatorname{dist}(C ; .)\left(u_{0}\right)=\partial_{F} \operatorname{dist}(C ; .)\left(u_{0}\right) .
$$

(ii) If in addition $X$ is a reflexive Banach space, then

$$
N\left(C ; u_{0}\right)=N_{F}\left(C ; u_{0}\right) .
$$

Proof. (i) Consider any $u_{0}^{*} \in \partial \operatorname{dist}(C ;).\left(u_{0}\right)$. Then for every $\varepsilon>0$ there exists $\delta>0$ such that for every $v \in C \cap B\left(u_{0}, \delta\right)$ we have $\left\langle-u_{0}^{*}, v-u_{0}\right\rangle \geq-\varepsilon\left\|v-u_{0}\right\|$, since $0 \in \partial \operatorname{dist}(C ;).(v)$. This inequality entails that $u_{0}^{*} \in N_{F}\left(C ; u_{0}\right)$. Further, $\left\|u_{0}^{*}\right\| \leq 1$ because $u_{0}^{*} \in \partial \operatorname{dist}(C ;).\left(u_{0}\right)$. According to (2.7), $u_{0}^{*} \in \partial_{F} \operatorname{dist}(C ;).\left(u_{0}\right)$ and hence $\partial \operatorname{dist}(C ;).\left(u_{0}\right) \subset \partial_{F} \operatorname{dist}(C ;).\left(u_{0}\right)$. Since the inverse inclusion is always true, we obtain $\partial \operatorname{dist}(C ;).\left(u_{0}\right)=\partial_{F} \operatorname{dist}(C ;).\left(u_{0}\right)$.

(ii) Combining formulas (2.8) and (2.7) with the equality established in $(i)$ we obtain

$$
N\left(C ; u_{0}\right)=w^{*}-\operatorname{cl}\left(N_{F}\left(C ; u_{0}\right)\right) .
$$

By Proposition 3.1 in Bounkhel-Thibault [3], the convex set $N_{F}\left(C ; u_{0}\right)=\partial_{F} \psi_{C}\left(u_{0}\right)$ is closed in $X^{*}$ with respect to the norm topology. As $X$ is reflexive, $N_{F}\left(C ; u_{0}\right)$ is also weak star closed. Thus (3.7) becomes $N\left(C ; u_{0}\right)=N_{F}\left(C ; u_{0}\right)$.

The following proposition deals with the statements arising from (3.2), if one replaces the truncated Clarke normal cone $N^{r}(C ;$.$) by some notion of subdifferential$ of the distance function.

Proposition 3.9. Let $C$ be a closed subset of an Asplund space $X$ and $u_{0} \in C$. Then the following assertions are equivalent:

(a) (3.2) holds with $\partial_{F} \operatorname{dist}(C ;$.$) in place of N^{r}(C ;$.$) ;$

(b) (3.2) holds with $\partial_{L} \operatorname{dist}(C ;$.$) in place of N^{r}(C ;$.$) ;$

(c) (3.2) holds with $\partial \operatorname{dist}(C ;$.$) in place of N^{r}(C ;$.$) .$ 
Proof. The implication $(c) \Longrightarrow(a)$ is an immediate consequence of the inclusion $\partial_{F} \operatorname{dist}(C ;.) \subset \partial \operatorname{dist}(C ;$.$) . Let us prove the implication (a) \Longrightarrow(b)$. Fix $\varepsilon>0$ and choose $\delta>0$ such that for all $x, y \in C \cap B\left(u_{0} ; \delta\right), x^{*} \in \partial_{F} \operatorname{dist}(C ;).(x)$ and $y^{*} \in \partial_{F} \operatorname{dist}(C ;).(y)$,

$$
\left\langle x^{*}-y^{*}, x-y\right\rangle \geq-\varepsilon\|x-y\| .
$$

Take any $u, v \in C \cap B\left(u_{0} ; \delta\right), u^{*} \in \partial_{L} \operatorname{dist}(C ;).(u)$ and $v^{*} \in \partial_{L} \operatorname{dist}(C ;).(v)$. By Proposition 3.5, there are $u_{n} \rightarrow_{C} u, v_{n} \rightarrow_{C} v,\left(u_{n}^{*}\right)$ and $\left(v_{n}^{*}\right)$ converging weak star to $u^{*}$ and $v^{*}$, with $u_{n}^{*} \in \partial_{F} \operatorname{dist}(C ;).\left(u_{n}\right)$ and $v_{n}^{*} \in \partial_{F} \operatorname{dist}(C ;).\left(v_{n}\right)$. For $n$ large enough we have $u_{n}, v_{n} \in C \cap B\left(u_{0} ; \delta\right)$ and hence according to the above inequality

$$
\left\langle u_{n}^{*}-v_{n}^{*}, u_{n}-v_{n}\right\rangle \geq-\varepsilon\left\|u_{n}-v_{n}\right\| .
$$

Passing to the limit, we obtain

$$
\left\langle u^{*}-v^{*}, u-v\right\rangle \geq-\varepsilon\|u-v\|,
$$

which translates assertion $(b)$.

It remains to show that $(b) \Longrightarrow(c)$. Let us observe that for each $\varepsilon>0$ and for fixed $u, v \in C \cap B\left(u_{0} ; \delta\right)$, the set of all $\left(u^{*}, v^{*}\right) \in X^{*} \times X^{*}$ satisfying the inequality $(3.9)$ is convex and weak star closed in $X^{*} \times X^{*}$. Thus the result follows from the equality (2.9).

Remark 3.10. Let us note that the proof of $(a) \Longrightarrow(b)$ in the above proposition will not work if one considers (3.3) instead of (3.2), since (3.8) will fail in general.

We now characterize subsmoothness in terms of the Fréchet normal cone when $X$ is a reflexive Banach space. In the following proposition we assume that $U$ is an open subset of $X$ and $C \cap U \neq \emptyset$.

Theorem 3.11. Let $C$ be a closed subset of a reflexive Banach space $X$. Then the following assertions are equivalent:

(a) $C$ is subsmooth on $C \cap U$;

(b) (3.2) holds at every point of $C \cap U$ with $N_{L}^{r}(C ;$.$) in place of N^{r}(C ;$.$) ;$

(c) (3.2) holds at every point of $C \cap U$ with $N_{F}^{r}(C ;$.$) in place of N^{r}(C ;$.$) ;$

(d) (3.2) holds at every point of $C \cap U$ with $\partial \operatorname{dist}(C ;$.$) in place of N^{r}(C ;$.$) .$

Proof. With no loss of generality we may assume in the above statements that $r=1$. Furthermore, implications $(a) \Longrightarrow(b)$ and $(b) \Longrightarrow(c)$ are direct consequences of the inclusions

$$
N_{F}^{1}(C ; u) \subset N_{L}^{1}(C ; u) \subset N^{1}(C ; u) \text {, for all } u \in C .
$$

Implication $(c) \Longrightarrow(d)$ follows from (2.7) and Proposition $3.9(a) \Longrightarrow(c)$. So, it remains to prove $(d) \Longrightarrow(a)$. It follows from Lemma 3.8 that for all $u \in C \cap U$,

$$
\partial \operatorname{dist}(C ; .)(u)=\partial_{F} \operatorname{dist}(C ; .)(u) \quad \text { and } \quad N(C ; u)=N_{F}(C ; u) .
$$

Combining (2.7) with (3.10) we get $N^{1}(C ; u)=\partial \operatorname{dist}(C ;).(u)$, for all $u \in C \cap U$. This completes the proof.

Remark 3.12. It is easily seen that one can add, in the list of equivalences of Theorem 3.11, the same property $(d)$ with any other subdifferential that is situated between the Fréchet and the Clarke subdifferentials. 
3.4. Comparison with relevant concepts. We are ready to proceed to the links of the notions of subsmoothness and semi-subsmoothness to other known tangential concepts in the literature, as Shapiro's property or Lewis' near radiality. The definition of the latter, that we recall in the next definition, is based on the notion of Bouligand cone; see (2.3).

Definition 3.13 (30, [18]). Let $C$ be a non-empty closed subset of $X$. Then

(i) $C$ is said to satisfy the $k$-order Shapiro property $(k \in \mathbb{N})$ at $u_{0} \in C$, if for every $\varepsilon>0$ there exists $\delta>0$ such that for all $u_{1}, u_{2} \in C \cap B\left(u_{0}, \delta\right)$ one has

$$
\operatorname{dist}\left(K\left(C ; u_{1}\right) ; u_{2}-u_{1}\right) \leq \varepsilon\left\|u_{1}-u_{2}\right\|^{k} .
$$

(ii) $C$ is called nearly radial at $u_{0} \in C$ if for every $\varepsilon>0$ there exists $\delta>0$ such that for all $u \in C \cap B\left(u_{0}, \delta\right)$ one has

$$
\operatorname{dist}\left(K(C ; u) ; u_{0}-u\right) \leq \varepsilon\left\|u-u_{0}\right\|
$$

(that is, (3.11) holds for $k=1$ with $x^{\prime}=x_{0}$ ).

The following two lemmas will be used in the sequel. The first one, concerning the Clarke tangent cone, involves a standard argument which has already been used in the literature (see [25, Proposition 3.6] or [6, Theorem 5.1] e.g.). Here we provide a complete proof for the reader's convenience.

Lemma 3.14. Let $C$ be a non-empty closed set and $u \in C$. Then for every $x \in X$

$$
\operatorname{dist}(T(C ; u) ; x)=\sup _{u^{*} \in N^{1}(C ; u)}\left\langle u^{*}, x\right\rangle .
$$

Proof. Put $T=T(C ; u)$ and $N=N(C ; u)$. If $\psi_{T}$ denotes the indicator function of the set $T$, then its Fenchel conjugate is given by $\psi_{T}^{*}=\psi_{N}$. Set further $s(y)=\|y\|$ and let $\mathbb{B}$ denote the closed unit ball of $X^{*}$. Note that

$$
\operatorname{dist}(T(C ; u) ; x)=\inf _{y \in X}\left[s(y-x)+\psi_{T}(y)\right] .
$$

Then by the Fenchel duality theorem we get

$$
\operatorname{dist}(T(C ; u) ; x)=\sup _{y^{*} \in X^{*}}\left[\left\langle y^{*}, x\right\rangle-s^{*}\left(y^{*}\right)-\psi_{T}^{*}\left(y^{*}\right)\right] .
$$

Since $s^{*}\left(y^{*}\right)=\psi_{\mathbb{B}}\left(y^{*}\right)$ and $\psi_{T}^{*}\left(y^{*}\right)=\psi_{N}\left(y^{*}\right)$, we conclude that

$$
\operatorname{dist}(T(C ; u) ; x)=\sup _{u^{*} \in N^{1}(C ; u)}\left\langle u^{*}, x\right\rangle .
$$

This finishes the proof.

The following result deals with the Bouligand tangent cone in finite dimensions.

Lemma 3.15. Assume that $X$ is a finite-dimensional space. Then

$$
\lim _{u \rightarrow C} \operatorname{dist}\left(K\left(C ; u_{0}\right) ; \frac{u-u_{0}}{\left\|u-u_{0}\right\|}\right)=0
$$

(where $\left.u \neq u_{0}\right)$.

Proof. Suppose that the above equality does not hold. Then there exist $\varepsilon>0$ and a sequence $u_{n} \rightarrow_{C} u_{0}\left(\right.$ with $\left.u_{n} \neq u_{0}\right)$ such that for all $n \geq 1$

$$
\operatorname{dist}\left(K\left(C ; u_{0}\right) ; \frac{u_{n}-u_{0}}{\left\|u_{n}-u_{0}\right\|}\right) \geq \varepsilon \text {. }
$$


Since $X$ is a finite-dimensional space, we may suppose (extracting a subsequence if necessary) that $\left\{\frac{u_{n}-u_{0}}{\left\|u_{n}-u_{0}\right\|}\right\}_{n \geq 1}$ converges to some vector $d \in X$. By the definition of the Bouligand tangent cone (see (2.3) $)$, it is easily seen that $d \in K\left(C ; u_{0}\right)$. But passing to the limit in (3.14) we obtain

$$
\operatorname{dist}\left(K\left(C ; u_{0}\right) ; d\right) \geq \varepsilon,
$$

which contradicts $d \in K\left(C ; u_{0}\right)$. The proof is complete.

In the following result, we make a comparison of the concepts in Definition 3.13 with the concepts of subsmoothness and semi-subsmoothness of sets. In the statements below, we suppose that $C$ is a closed set and $U$ is an open subset of $X$ such that $C \cap U \neq \emptyset$.

Theorem 3.16. (a) $C$ is subsmooth on $C \cap U$ if, and only if, it is tangentially regular on $C \cap U$ and satisfies the first order Shapiro property on $C \cap U$.

(b) If $X$ is reflexive, then $C$ is subsmooth on $C \cap U$ if, and only if, it satisfies the first order Shapiro property on $C \cap U$.

(c) If $C$ is semi-subsmooth on $C \cap U$, then it is tangentially regular on $C \cap U$ and nearly radial on $C \cap U$. The converse also holds whenever $X$ is a finite-dimensional space.

Proof. (a) To prove the "necessity" part, let us suppose that $C$ is subsmooth on $C \cap U$. Then by Proposition 3.4 (ii) and (iii), $C$ is also tangentially regular at every point in $C \cap U$. Let us fix any $u_{0} \in C \cap U$ and let us show that $C$ satisfies the first order Shapiro property at $u_{0}$. According to Definition [3.1, for every $\varepsilon>0$ there exists $\delta>0$ such that

$$
\left\langle u_{1}^{*}-u_{2}^{*}, u_{1}-u_{2}\right\rangle \geq-\varepsilon\left\|u_{1}-u_{2}\right\|
$$

for all $u_{i} \in C \cap B\left(u_{0}, \delta\right)$ and all $u_{i}^{*} \in N^{1}\left(C ; u_{i}\right)$ for $i \in\{1,2\}$. Taking $u_{2}^{*}=0$, the above yields

$$
\sup _{u_{1}^{*} \in N^{1}\left(C ; u_{1}\right)}\left\langle u_{1}^{*}, u_{2}-u_{1}\right\rangle \leq \varepsilon\left\|u_{1}-u_{2}\right\| .
$$

Moreover, from the tangential regularity we have $T\left(C ; u_{1}\right)=K\left(C ; u_{1}\right)$. Combining this with relations (3.15) and (3.13) we obtain

$$
\operatorname{dist}\left(K\left(C ; u_{1}\right) ; u_{2}-u_{1}\right) \leq \varepsilon\left\|u_{1}-u_{2}\right\|,
$$

that is, $C$ satisfies the first order Shapiro property at $u_{0}$.

Conversely, to prove the "sufficiency" part, let us assume that $C$ is tangentially regular and satisfies the first order Shapiro property at each point of $C \cap U$. Take any $u_{0} \in C \cap U$. We must show that $C$ is subsmooth at $u_{0}$. To this end, let $\varepsilon>0$. Then by Definition [3.13 $(i)$ (for $k=1$ ), there exists $\delta>0$ such that $B\left(u_{0}, \delta\right) \subset U$ and for all $u_{i} \in C \cap B\left(u_{0}, \delta\right), i \in\{1,2\}$,

$$
\max \left\{\operatorname{dist}\left(K\left(C ; u_{1}\right) ; u_{2}-u_{1}\right), \operatorname{dist}\left(K\left(C ; u_{2}\right) ; u_{1}-u_{2}\right)\right\} \leq \frac{\varepsilon}{2}\left\|u_{1}-u_{2}\right\| .
$$

Since $K\left(C ; u_{i}\right)=T\left(C ; u_{i}\right)$ for $i \in\{1,2\}$, in view of Lemma 3.14 we get

$$
\max \left\{\sup _{u_{1}^{*} \in N^{1}\left(C ; u_{1}\right)}\left\langle u_{1}^{*}, u_{2}-u_{1}\right\rangle, \sup _{u_{2}^{*} \in N^{1}\left(C ; u_{2}\right)}\left\langle u_{2}^{*}, u_{1}-u_{2}\right\rangle\right\} \leq \frac{\varepsilon}{2}\left\|u_{1}-u_{2}\right\| .
$$


The above easily yields that for all $u_{i}^{*} \in N^{1}\left(C ; u_{i}\right), i \in\{1,2\}$, we have

$$
\left\langle u_{1}^{*}-u_{2}^{*}, u_{1}-u_{2}\right\rangle \geq-\varepsilon\left\|u_{1}-u_{2}\right\|,
$$

which shows that $C$ is subsmooth at $x_{0}$.

(b) Assume that $X$ is reflexive. Taking (a) into account, we only have to prove the "sufficiency" part. To this end, let us suppose that $C$ satisfies the first order Shapiro property on $C \cap U$ and let us show that $C$ is subsmooth there. In view of Theorem $3.11(c) \Longrightarrow(a)$, it suffices to show that the truncated Fréchet normal cone $N_{F}^{1}(C ;$.$) satisfies (3.2) at every point of C \cap U$. So, let us fix any $u_{0} \in C \cap U$ and $\varepsilon>0$. As in the proof of the "sufficiency" part of $(a)$, there exists $\delta>0$ such that (3.16) holds. Let us consider any $u_{i} \in C \cap B\left(u_{0}, \delta\right)$ and $u_{i}^{*} \in N_{F}^{1}\left(C ; u_{i}\right), i \in\{1,2\}$. Then, according to (3.16), we may choose $v_{i} \in K\left(C ; u_{i}\right)$ and $e_{i} \in X$ with $i \in\{1,2\}$ such that

$$
u_{2}-u_{1}=v_{1}+e_{1} \quad \text { and } \quad u_{1}-u_{2}=v_{2}+e_{2},
$$

with $\left\|e_{i}\right\| \leq \frac{\varepsilon}{2}\left\|u_{1}-u_{2}\right\|$ for $i \in\{1,2\}$. Using the definition of the Fréchet normal cone and the definition of the Bouligand tangent cone, it is not difficult to see that $\left\langle u_{i}^{*}, v_{i}\right\rangle \leq 0$, for $i \in\{1,2\}$. Further, since $\left\|u_{i}^{*}\right\| \leq 1$, for $i \in\{1,2\}$, it follows that

$$
\left\langle u_{1}^{*}, u_{1}-u_{2}\right\rangle \geq-\frac{\varepsilon}{2}\left\|u_{1}-u_{2}\right\| \quad \text { and } \quad\left\langle u_{2}^{*}, u_{2}-u_{1}\right\rangle \geq-\frac{\varepsilon}{2}\left\|u_{1}-u_{2}\right\| .
$$

Putting these inequalities together, we obtain (3.2). This completes the proof.

(c) Let us assume that $C$ is semi-subsmooth on $C \cap U$. By Proposition 3.4 (iii), $C$ is tangentially regular at any point in $C \cap U$. To prove that $C$ is nearly radial at a point $u_{0} \in C \cap U$ one proceeds in the same way as in the above proof of the "necessity" part of $(a)$, by setting $u_{2}=u_{0}$ (and $u_{1}=u$ ) to conclude that (3.12) holds.

Let us now suppose that $X$ is a finite-dimensional space and that $C$ is tangentially regular and nearly radial at each point in $C \cap U$. Let us fix any $u_{0} \in C \cap U$, and let us show that (3.3) holds. To this end, take any $\varepsilon>0$. By Definition 3.13( $i i)$, Lemma 3.14 (relation (3.13)) and the tangential regularity on $C \cap U$ we conclude that for some $\delta>0$ such that $B\left(u_{0}, \delta\right) \subset U$ and for all $u \in C \cap B\left(u_{0}, \delta\right)$

$$
\sup _{u^{*} \in N^{1}(C ; u)}\left\langle u^{*}, u_{0}-u\right\rangle \leq \frac{\varepsilon}{2}\left\|u-u_{0}\right\| .
$$

By Lemma 3.15, we may also suppose that for every $u \in C \cap B\left(u_{0}, \delta\right)$ with $u \neq u_{0}$

$$
\operatorname{dist}\left(K\left(C ; u_{0}\right) ; \frac{u-u_{0}}{\left\|u-u_{0}\right\|}\right)<\frac{\varepsilon}{2}, \quad \text { i.e., } \quad \operatorname{dist}\left(K\left(C ; u_{0}\right) ; u-u_{0}\right) \leq \frac{\varepsilon}{2}\left\|u-u_{0}\right\| .
$$

Since $K\left(C ; u_{0}\right)=T\left(C ; u_{0}\right)$, using Lemma 3.14 again we obtain

$$
\sup _{u_{0}^{*} \in N^{1}\left(C ; u_{0}\right)}\left\langle u_{0}^{*}, u-u_{0}\right\rangle \leq \frac{\varepsilon}{2}\left\|u-u_{0}\right\| .
$$

From (3.17) and (3.19) we easily conclude that $C$ is semi-subsmooth at $u_{0}$.

The following corollary concerns the tangential regularity of sets satisfying the first-order Shapiro property.

Corollary 3.17. In a reflexive Banach space, every set $C$ satisfying the first-order Shapiro property on $C \cap U$ is normally Fréchet regular and tangentially regular on $C \cap U$. 
Proof. The corollary follows directly from $(b)$ in Theorem 3.16] and from Proposition 3.4.

Remark 3.18. Tangential regularity cannot be deduced from near radiality. Indeed, let $C=$ epi $f \subset \mathbb{R}^{2}$, where $f: \mathbb{R} \rightarrow \mathbb{R}$ is given by $f(x)=-|x|$ for all $x \in \mathbb{R}$. Then $C$ is nearly radial at every point of $C$, but it is not tangentially regular at $u_{0}=(0,0)$.

Let us finish this section by summarizing the relations among the considered notions. Some of them were already known in the literature. Any implication which is not explicitly stated in the diagram is not in general true.

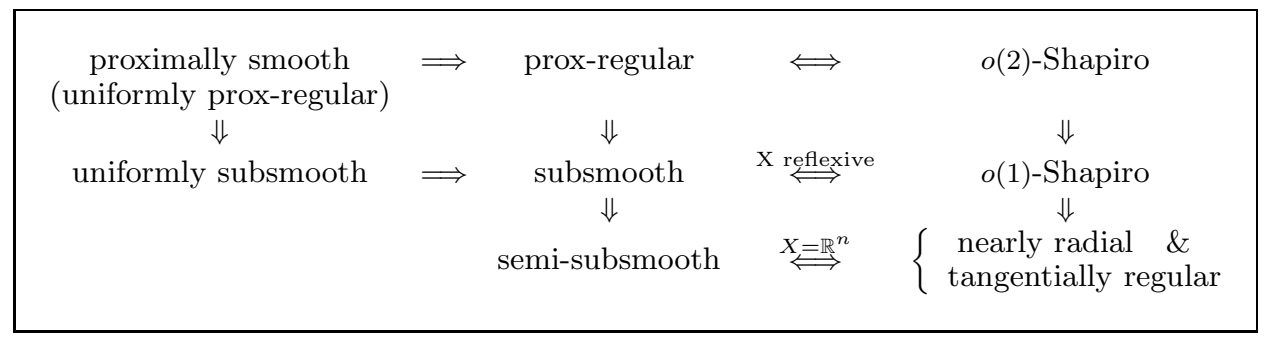

\section{Main Results: Links Between Set and functional Notions}

In this section we shall establish links among epi-Lipschitz sets and functional notions. In particular, we shall show that epi-Lipschitz sets are subsmooth if, and only if, they can be represented by approximately convex functions (see Corollary 4.17). In finite dimensions these functions correspond to the lower- $\mathrm{C}^{1}$ property (see [9]).

Let us recall the functional notions that we are going to evoke in the sequel. We denote by $T: X \rightrightarrows X^{*}$ a multivalued operator with domain $\operatorname{dom} T:=\{x \in X$ : $T(x) \neq \emptyset\}$.

4.1. Hypomonotonicity and weak convexity. We recall from 28 that $T$ is hypomonotone at $x_{0}$ if there exists $\sigma, \delta>0$ such that for all $x_{1}, x_{2} \in B\left(x_{0}, \delta\right) \cap \operatorname{dom} T$ and all $x_{i}^{*} \in T\left(x_{i}\right), i \in\{1,2\}$, one has

$$
\left\langle x_{1}^{*}-x_{2}^{*}, x_{1}-x_{2}\right\rangle \geq-\sigma\left\|x_{1}-x_{2}\right\|^{2} .
$$

The operator $T$ is called hypomonotone on $X$ (respectively, on $A \subset X$ ) if it is hypomonotone at every point of $X$ (respectively, of $A$ ).

It follows from this definition that for a non-empty closed set $C$ of a Hilbert space $X$ one has:

- $C$ is prox-regular if, and only if, $N^{r}(C,$.$) is hypomonotone for any r>0$.

Hypomonotonicity is tidily related to weakly convex functions (33]), or in finite dimensions to lower- $\mathrm{C}^{2}$ functions ([27], [5] e.g.). The exact relation in Hilbert spaces is given in the next theorem. The equivalence of $(i i)$ and (iii) in Hilbert spaces can be found in [2, Proposition 3.6], as well as the equivalence with the decomposability to the difference of a convex function and a multiple of the square of the norm. In the present context, we establish a direct proof of the equivalence between $(i)$ and (ii). Before giving the proof, let us consider a pointwise refinement of the definition 
(1.1): A function $f: X \rightarrow \mathbb{R} \cup\{+\infty\}$ is called weakly convex at $x_{0} \in \operatorname{dom} f$ if there exists $\sigma, \delta>0$ such that for all $x, y \in B\left(x_{0}, \delta\right)$ and $t \in[0,1]$,

$$
f(t x+(1-t) y) \leq t f(x)+(1-t) f(y)+\sigma t(1-t)\|x-y\|^{2} .
$$

Clearly, $f$ is weakly convex if, and only if, it is weakly convex at every $x_{0} \in \operatorname{dom} f$. Note also that the weak convexity of $f$ at a point $x_{0}$ implies its weak convexity in a neighbourhood of $x_{0}$ (however, with different values for the parameters $\sigma$ and $\delta$ in general). Let us finally mention that similar to the convex functions, weakly convex functions are locally Lipschitz in the interior of their domain $\operatorname{int}(\operatorname{dom} f)$.

In the following result, $f$ will merely be assumed lower semicontinuous. We recall ([27]) that in this case the Clarke subdifferential $\partial f$ is given by the formula (2.4), with $f^{0}\left(x_{0} ;.\right)$ being replaced by the Rockafellar generalized directional derivative $f^{\uparrow}\left(x_{0} ;.\right)$, where

$$
f^{\uparrow}\left(x_{0} ; u\right):=\sup _{\delta>0} \limsup _{\substack{x \rightarrow x_{f} x_{0} \\ t \succ^{+}}} \inf _{w \in B(u, \delta)} \frac{f(x+t w)-f(x)}{t} .
$$

Note that in the above formula, $\sup _{\delta>0}$ can be replaced by $\lim _{\delta \backslash 0^{+}}$.

Theorem 4.1. Let $X$ be a Hilbert space, let $f: X \rightarrow \mathbb{R} \cup\{+\infty\}$ be a proper lower semicontinuous function and let $x_{0} \in \operatorname{dom} f$. The following statements are equivalent:

(i) $f$ is weakly convex at $x_{0}$;

(ii) $\partial f$ is hypomonotone at $x_{0}$;

(iii) there exists $\sigma>0$ and $\delta>0$ such that for all $x, y \in B\left(x_{0}, \delta\right)$, and all $x^{*} \in \partial f(x)$,

$$
f(y) \geq f(x)+\left\langle x^{*}, y-x\right\rangle-\sigma\|y-x\|^{2} .
$$

If $X=\mathbb{R}^{n}$ and $x_{0} \in \operatorname{int}(\operatorname{dom} f)$, then the above statements are also equivalent to

(iv) $f$ is lower- $C^{2}$ at $x_{0}$.

Proof. $(i) \Longrightarrow($ iii $)$. Let $\sigma>0$ and $\delta_{0}>0$ be such that for all $x, y \in B\left(x_{0}, \delta_{0}\right)$ and $t \in[0,1]$, one has

$$
f(t x+(1-t) y) \leq t f(x)+(1-t) f(y)+\sigma t(1-t)\|x-y\|^{2} .
$$

Fix any $x, y \in B\left(x_{0}, \delta_{0} / 8\right)$. Also fix any $0<\delta<\delta_{0}$. Then for all $z \in B(x, \delta / 4) \cap$ $\operatorname{dom} f, v \in X$ with $0<\|v\|<\delta_{0} / 2$ and $\left.t \in\right] 0,1[$ we obtain from (4.5)

$$
f(z+t v)=f((1-t) z+t(z+v)) \leq t f(z+v)+(1-t) f(z)+\sigma t(1-t)\|v\|^{2},
$$

from which we infer that

$$
\frac{f(z+t v)-f(z)}{t} \leq f(z+v)-f(z)+\sigma(1-t)\|v\|^{2} .
$$

In particular for $v^{\prime}=y-z$ we have $v^{\prime} \in B(y-x, \delta / 4)$ and $\left\|v^{\prime}\right\|<\delta_{0} / 2$, hence using the above we obtain

$$
\inf _{w \in B(y-x, \delta)} \frac{f(z+t w)-f(z)}{t} \leq f(y)-f(z)+\sigma(1-t)\|y-z\|^{2} .
$$

It follows that

$$
\limsup _{\substack{z \rightarrow f x \\ t \backslash 0^{+}}} \inf _{w \in B(y-x, \delta)} \frac{f(z+t w)-f(z)}{t} \leq f(y)-f(x)+\sigma\|y-x\|^{2},
$$


and hence, passing to the limit for $\delta \searrow 0^{+}$one gets according to (4.3)

$$
f^{\uparrow}\left(x_{0} ; y-x\right) \leq f(y)-f(x)+\sigma\|y-x\|^{2} .
$$

This yields that (iii) holds (with $\delta^{\prime}=\delta_{0} / 8$ ).

$($ iii $) \Longrightarrow($ ii $)$. Let $\sigma>0$ and $\delta>0$ be such that (4.4) holds with $\sigma / 2$. Then for any $x, y \in B\left(x_{0}, \delta\right), x^{*} \in \partial f(x)$ and $y^{*} \in \partial f(y)$, writing (4.4) one time with $x^{*} \in \partial f(x)$ and another time with $y^{*} \in \partial f(y)$ and adding by parts, one immediately obtains (4.1).

$(i i) \Longrightarrow(i)$. In finite dimensions this corresponds to implication $(b) \Longrightarrow(c)$ of 28, Theorem 6]. However, the same proof can be adopted in Hilbert spaces. We recall it for completeness: Let us fix $\sigma>0$ and $\delta>0$ such that (4.1) holds for the operator $\partial f$ on $B\left(x_{0}, \delta\right)$. For every $x \in X$, let us define $g(x)=f(x)+(\sigma / 2)\|x\|^{2}$ and let us note that $\partial g=\partial f+\sigma I$ (where $I$ is the identity operator). It follows from (4.1) that $\partial g$ is monotone on $B\left(x_{0}, \delta\right)$. Thus, the lower semicontinuous function $g+\psi_{B\left[x_{0}, \delta_{1}\right]}$ is convex (see [8], [1]) for every $0<\delta_{1}<\delta$, and $f$ can be expressed as the difference of the convex function $g+\psi_{B\left(x_{0}, \delta\right)}$ and the convex quadratic function $h(x)=(\sigma / 2)\|x\|^{2}$ on $B\left(x_{0}, \delta_{1}\right)$. In view of [33. Proposition 4.3] (which remains true in infinite dimensions) $f$ satisfies (4.2).

The equivalence $(i i) \Longleftrightarrow(i v)$ in finite dimensions has been established in 28 .

We must mention that the equivalence between the assertions $(i),(i i)$, and $(i i i)$ in finite dimensions can be found in [5]. Lower- $\mathrm{C}^{2}$ functions in Hilbert spaces (as well as their connection with lower- $\mathrm{T}^{2}$ functions) are studied in detail in [24].

4.2. Submonotonicity and approximate convexity. A weaker notion to hypomonotonicity is the concept of submonotonicity (see [31], [10]).

Definition 4.2. An operator $T: X \rightrightarrows X^{*}$ is called

(i) submonotone at $x_{0} \in X$ if for every $\varepsilon>0$ there exists $\delta>0$ such that for all $x_{1}, x_{2} \in B\left(x_{0}, \delta\right) \cap \operatorname{dom} T$ and all $x_{i}^{*} \in T\left(x_{i}\right), i \in\{1,2\}$, one has

$$
\left\langle x_{1}^{*}-x_{2}^{*}, x_{1}-x_{2}\right\rangle \geq-\varepsilon\left\|x_{1}-x_{2}\right\| ;
$$

(ii) submonotone on $X$ (respectively, on $A \subset X$ ) if it is submonotone at any point of $X$ (respectively, of $A$ ).

In view of the previous definition, Definition 3.1 can be restated as follows:

- $C$ is subsmooth if, and only if, $N^{r}(C ;$.) is submonotone for any $r>0$.

Let us recall from the Introduction the following definition.

Definition $4.3(23])$. A function $f: X \rightarrow \mathbb{R} \cup\{+\infty\}$ is called

(i) approximately convex at $x_{0}$ if for every $\varepsilon>0$ there exists $\delta>0$ such that, for all $x, y \in B\left(x_{0}, \delta\right)$ and $t \in[0,1]$, one has

$$
f(t x+(1-t) y) \leq t f(x)+(1-t) f(y)+\varepsilon t(1-t)\|x-y\| ;
$$

(ii) approximately convex if it is approximately convex at every point of $\operatorname{dom} f$.

As in the case of convex or weakly convex functions, every approximate convex function is locally Lipschitz on $\operatorname{int}(\operatorname{dom} f)($ see [23, Proposition 3.2]). We also refer the reader to Rolewicz [29] for similar convex-like functions.

In the sequel we shall give a characterization analogous to Theorem4.1 in infinite dimensions. Before, let us state the following lemma, which is inspired from the 
proof of [31, Theorem 3.9]. We denote by $C_{*}^{1}\left(\mathbb{R}, \mathbb{R}_{+}\right)$the set of all non-negative continuously differentiable functions $a: \mathbb{R} \longrightarrow \mathbb{R}_{+}$satisfying $a(0)=a^{\prime}(0)=0$.

Lemma 4.4. Let $T: X \rightrightarrows X^{*}$ be a multivalued operator, let $f: X \rightarrow \mathbb{R} \cup\{+\infty\}$ be a proper function and $x_{0} \in \operatorname{cl}(\operatorname{dom} f)$. The following assertions are equivalent:

(i) For every $\varepsilon>0$ there exists $\delta>0$ such that for all $x, y \in B\left(x_{0}, \delta\right) \cap \operatorname{dom} f$, $x^{*} \in T(x)$,

$$
f(y) \geq f(x)+\left\langle x^{*}, y-x\right\rangle-\varepsilon\|y-x\| ;
$$

(ii) there exists $\delta>0$ and an even function $a \in C_{*}^{1}\left(\mathbb{R}, \mathbb{R}_{+}\right)$such that for all $x, y \in B\left(x_{0}, \delta\right) \cap \operatorname{dom} f, x^{*} \in T(x)$,

$$
f(y) \geq f(x)+\left\langle x^{*}, y-x\right\rangle-a(\|y-x\|) .
$$

Proof. $(i) \Longrightarrow(i i)$. For every $x, y \in \operatorname{dom} f$ and $x^{*} \in X^{*}$ we set

$$
\sigma\left(x, y, x^{*}\right)=\left\{\begin{array}{cc}
{\left[f(y)-f(x)-\left\langle x^{*}, y-x\right\rangle\right]\|y-x\|^{-1}} & \text { if } x \neq y \\
0 & \text { if } x=y
\end{array}\right.
$$

and for every $t>0$ we define

$$
\varphi_{1}(t)=\inf \left\{\sigma\left(x, y, x^{*}\right): x, y \in B\left(x_{0}, t\right) \cap \operatorname{dom} f, x^{*} \in T(x)\right\},
$$

with the obvious convention $\varphi_{1}(t)=+\infty$ whenever $T(x)=\emptyset$. We also set $\varphi_{1}(0)=$ 0 .

Let us assume that assertion $(i)$ holds. Then for every $\varepsilon>0$ there exists $\delta>0$ such that for all $t \in[0, \delta)$ we have

$$
\varphi_{1}(t) \geq-\varepsilon .
$$

It follows that the function $\varphi:[0,+\infty) \rightarrow \mathbb{R}$ defined by

$$
\varphi(t)=\max \left\{-\varphi_{1}(t), 0\right\}
$$

is continuous at $t=0$. Applying [31, Lemma 3.7] to the function $\varphi$ we conclude that for some $\delta_{1}>0$ and some continuously differentiable function $a_{1}:\left[0, \delta_{1}\right] \longrightarrow \mathbb{R}$ with $a_{1}(0)=a_{1}^{\prime}(0)=0$ we have

$$
a_{1}(t) \geq t \varphi(t), \forall t \in\left[0, \delta_{1}\right] .
$$

Clearly, $a_{1}(t) \geq 0$, for all $t \in\left[0, \delta_{1}\right]$. We extend the function $a_{1}$ to $[0,+\infty)$ by defining $\tilde{a}_{1}(t)=a_{1}(t)$ if $t \in\left[0, \delta_{1}\right]$ and $\tilde{a}_{1}(t)=a_{1}\left(\delta_{1}\right)+a_{1}^{\prime}\left(\delta_{1}\right)\left(t-\delta_{1}\right)$ if $t>\delta_{1}$, and we define $a: \mathbb{R} \longrightarrow \mathbb{R}$ in the following way:

$$
a(t)=\left\{\begin{array}{cc}
\tilde{a}_{1}(t), & \text { if } t \geq 0, \\
\tilde{a}_{1}(-t), & \text { if } t<0 .
\end{array}\right.
$$

Then $a$ is an even, non-negative, continuously differentiable function with $a(0)=$ $a^{\prime}(0)=0$ and satisfying relation (4.13). We set $\delta=\delta_{1} / 2$. Let us verify that relation (4.9) holds for any $x, y \in B\left(x_{0}, \delta\right) \cap \operatorname{dom} f$ and $x^{*} \in T(x)$. This is trivially the case if $x=y$. Otherwise, we set $t=\|y-x\|>0$. Since $t \leq \delta_{1}$, combining formulas (4.11), (4.12) and (4.13) we infer

$$
\sigma\left(x, y, x^{*}\right) \geq \varphi_{1}(t) \geq-\varphi(t) \geq-\frac{a(t)}{t}:=-\frac{a(\|y-x\|)}{\|y-x\|},
$$

which yields (4.9).

$($ ii $) \Longrightarrow(i)$. Let $\tilde{\delta}>0$ and $a$ be such that relation (4.9) holds for any $x, y \in$ $B\left(x_{0}, \tilde{\delta}\right) \cap \operatorname{dom} f$ and $x^{*} \in T(x)$. Since the function $a$ is derivable at $t=0$ with 
$a(0)=0$ and $a^{\prime}(0)=0$, for every $\varepsilon>0$ there exists $\delta_{1}>0$ such that $a(t)<\varepsilon t$ for all $\left.t \in] 0, \delta_{1}\right]$. Set $\delta=\min \left\{\tilde{\delta}, \delta_{1} / 2\right\}$. Then for every $x, y \in B\left(x_{0}, \delta\right)$, one has $a(\|y-x\|) \leq \varepsilon\|y-x\|$. Consequently, relation (4.8) follows directly from (4.9).

Theorem 4.5. Let $f: X \rightarrow \mathbb{R} \cup\{+\infty\}$ be a proper function. Suppose that $f$ is locally Lipschitz on $U$, where $U$ is an open subset of $\operatorname{dom} f$ and let $x_{0} \in U$. Then the following statements are equivalent:

(i) $f$ is approximately convex at $x_{0}$;

(ii) $\partial f$ is submonotone at $x_{0}$;

(iii) for every $\varepsilon>0$ there exists $\delta>0$ such that for all $x, y \in B\left(x_{0}, \delta\right)$, and all $x^{*} \in \partial f(x)$,

$$
f(y) \geq f(x)+\left\langle x^{*}, y-x\right\rangle-\varepsilon\|y-x\| ;
$$

(iv) there exists $\delta>0$ and an even function $a \in C_{*}^{1}\left(\mathbb{R}, \mathbb{R}_{+}\right)$such that for all $x, y \in B\left(x_{0}, \delta\right)$, and all $x^{*} \in \partial f(x)$,

$$
f(y) \geq f(x)+\left\langle x^{*}, y-x\right\rangle-a(\|y-x\|) .
$$

If $X=\mathbb{R}^{n}$ the statements $(\mathrm{i}),(\mathrm{ii}),(\mathrm{iii})$ and (iv) above are also equivalent to

(v) $f$ is lower- $C^{1}$ at $x_{0}$.

Proof. The equivalences $(i) \Longleftrightarrow(i i) \Longleftrightarrow$ (iii) have been established in [9, Theorem 1], while the equivalence of (iii) with $(i v)$ is a direct consequence of Lemma 4.4. Finally, in finite dimensions, the equivalence between $(v)$ and $(i i)$ has been established in [31. Let us give a simpler proof of $(i v) \Longrightarrow(v)$. So, let us assume that $X=\mathbb{R}^{n}$ and for any fixed $x_{0} \in U$ let us choose $\delta>0$ such that $B\left[x_{0} ; \delta\right] \subset U$ and (4.15) holds. Since $\partial f$ is locally bounded and has a closed graph $\operatorname{gph}(\partial f)$, and since the closed ball $B\left[x_{0}, \delta\right]$ is compact, we conclude that the set $S\left(x_{0}, \delta\right)=\left\{\left(x, x^{*}\right) \in \operatorname{gph}(\partial f): x \in B\left[x_{0}, \delta\right]\right\}$ is also compact. It follows directly from (4.15) that for all $y \in B\left[x_{0}, \delta\right]$

$$
f(y)=\max _{s \in S\left(x_{0}, \delta\right)} F(y, s)
$$

where $F\left(y,\left(x, x^{*}\right)\right)=f(x)+\left\langle x^{*}, y-x\right\rangle-a(\|y-x\|)$. Since $a \in C_{*}^{1}\left(\mathbb{R}, \mathbb{R}_{+}\right)$, the function $F(\cdot, \cdot)$ is also continuously differentiable with respect to the first variable. It follows from (4.16) that $f$ is lower- $C^{1}$ at $x_{0}$.

Remark 4.6. By fixing $\varepsilon_{0}>0$ in Definition $4.2(i)$ (respectively, in Definition 4.3(i)) and assuming the existence of some $\delta>0$ such that (4.6) (respectively, (4.7)) holds, one can define the notions of $\varepsilon_{0}$-submonotonicity (respectively, $\varepsilon_{0}$-approximate convexity). Thus, an operator $T$ is submonotone (respectively, a function $f$ is approximately convex) at $x_{0}$ if it is $\varepsilon$-submonotone (respectively, $\varepsilon$-approximately convex) at $x_{0}$ for every $\varepsilon>0$. It is easily seen that an $\varepsilon_{0}$-version of the equivalences $(i) \Longleftrightarrow(i i) \Longleftrightarrow($ iii $)$ of Theorem 4.5 can be established.

4.3. Semi-submonotonicity and semismoothness. The definition of submonotone operator (Definition [4.2(ii)) has been introduced by Spingarn [31] in finite dimensions. Let us mention that Spingarn employed the term "strict submonotonicity" for this notion, and reserved the term "submonotonicity" for the more restrictive one where (4.6) holds only if $x_{2}=x_{0}$. To distinguish between these two notions we propose for the latter the term "semi-submonotonicity". 
Definition 4.7. An operator $T: X \rightrightarrows X^{*}$ is called

(i) semi-submonotone at $x_{0} \in X$, if for every $\varepsilon>0$, there exists $\delta>0$, such that, for all $x \in B\left(x_{0}, \delta\right) \cap \operatorname{dom} T$, all $x^{*} \in T(x)$, and all $x_{0}^{*} \in T\left(x_{0}\right)$ one has

$$
\left\langle x^{*}-x_{0}^{*}, x-x_{0}\right\rangle \geq-\varepsilon\left\|x-x_{0}\right\| ;
$$

(ii) semi-submonotone, if it is semi-submonotone at all $x \in X$.

Similar to sections 4.1 and 4.2 above, given a closed set $C$ and $u_{0} \in C$ one has (see Definition 3.2

- $C$ is semi-subsmooth if, and only if, $N^{r}(C ;$.$) is semi-submonotone for any$ $r>0$.

We shall now introduce a new class of functions, that we call "semismooth", by weakening the definition of approximate convexity.

Definition 4.8. A function $f: X \rightarrow \mathbb{R} \cup\{+\infty\}$ is called

(i) semismooth at $x_{0}$ if for every $\varepsilon>0$ there exists $\delta>0$ such that, for all $x \in B\left(x_{0}, \delta\right)$ and $\left.t \in\right] 0,1[$, one has

$$
f\left(t x+(1-t) x_{0}\right) \leq t f(x)+(1-t) f\left(x_{0}\right)+\varepsilon t(1-t)\left\|x-x_{0}\right\| ;
$$

(ii) semismooth if it is semismooth at every point of $\operatorname{dom} f$.

We must mention that a different notion of semismoothness had been previously introduced by Mifflin in 20] in finite dimensions (see also 7], for an extension in infinite dimensions). To avoid confusion, let us refer to this latter notion as $M$ semismoothness. According to that definition, a function $f$ is called $M$-semismooth if for every sequence $\left\{x_{n}\right\}_{n} \subset X$, every $x \in X, e \in S_{X}$ and every $\left\{x_{n}^{*}\right\}_{n} \subset X^{*}$ such that $x_{n}^{*} \in \partial f\left(x_{n}\right)$ and $\left(x_{n}\right) \stackrel{e}{\rightarrow} x$, we get $\left\langle x_{n}^{*}, e\right\rangle \rightarrow f^{\prime}(x ; e)$, where

$$
\left(x_{n}\right) \stackrel{e}{\rightarrow} x \Longleftrightarrow \lim _{n \rightarrow+\infty} x_{n}=x \quad \& \quad \lim _{n \rightarrow+\infty}\left\|\frac{x_{n}-x}{\left\|x_{n}-x\right\|}-e\right\|=0
$$

and where $f^{\prime}(x ; e)$ denotes the usual directional derivative given by

$$
f^{\prime}(x ; e)=\lim _{t \rightarrow 0^{+}} \frac{f(x+t e)-f(x)}{t} .
$$

We note that none of the aforementioned notions of semismoothness implies the directional regularity of the function: Indeed, the function $f: \mathbb{R} \rightarrow \mathbb{R}$ with $f(x)=$ - $|x|$ is both semismooth and $M$-semismooth, but it is clearly not d-regular at $x_{0}=0$.

In the following proposition we establish links among the above notions as well as with the notion of semi-subsmoothness.

Theorem 4.9. Let $f: X \rightarrow \mathbb{R} \cup\{+\infty\}$ be a proper function. Suppose that $f$ is locally Lipschitz on $U$, where $U$ is an open subset of $\operatorname{dom} f$ and consider the following statements:

(i) $f$ is semismooth and d-regular on $U$;

(ii) $\partial f$ is semi-submonotone on $U$;

(iii) for all $x_{0} \in U$ and $\varepsilon>0$ there exists $\delta>0$ such that for all $x, y \in B\left(x_{0}, \delta\right)$ such that either $x=x_{0}$ or $y=x_{0}$, and for all $x^{*} \in \partial f(x)$,

$$
f(y) \geq f(x)+\left\langle x^{*}, y-x\right\rangle-\varepsilon\|y-x\| ;
$$


(iv) $f$ is $M$-semismooth and d-regular on $U$.

Then $(i) \Longrightarrow($ iii $) \Longrightarrow(i i) \Longrightarrow(i v)$.

If $X=\mathbb{R}^{n}$, then all the above statements are equivalent.

Proof. (i) $\Longrightarrow$ (iii). Fix $x_{0} \in U$ and $\varepsilon>0$ and take $\delta>0$ such that (4.18) holds. Consider any $x \in B\left(x_{0}, \delta\right)$ and any $\left.t \in\right] 0,1\left[\right.$. Since $f\left(x_{0}+t\left(x-x_{0}\right)\right)=$ $f\left(t x+(1-t) x_{0}\right)$, relation (4.18) yields

$$
f\left(x_{0}+t\left(x-x_{0}\right)\right)-f\left(x_{0}\right) \leq t\left\{f(x)+f\left(x_{0}\right)+\varepsilon(1-t)\left\|x-x_{0}\right\|\right\} .
$$

Dividing by $t$ and taking the limit as $t \rightarrow 0^{+}$we obtain

$$
d^{-} f\left(x_{0} ; x-x_{0}\right) \leq f(x)-f\left(x_{0}\right)+\varepsilon\left\|x-x_{0}\right\| .
$$

Similarly for $s=(1-t)$ and $f\left(x+s\left(x_{0}-x\right)\right)=f\left((1-s) x+s x_{0}\right)$, applying (4.18) and proceeding as before we obtain formula (4.20) with $x$ and $x_{0}$ mutually changed. Since $f$ is d-regular, $d^{-} f(x ;)=.f^{0}(x ;$.$) and d^{-} f\left(x_{0} ;.\right)=f^{0}\left(x_{0} ;.\right)$. Thus, in view of (2.4), relation (4.19) follows.

(iii) $\Longrightarrow$ (ii). Fix $x_{0} \in U$ and $\varepsilon>0$. Then take $\delta>0$ such that (iii) holds with $\varepsilon / 2$. Consider any $x \in B\left(x_{0}, \delta\right)$. Applying now (4.19) one time for $y=x_{0}$ and another time for $x=x_{0}$ (and $y=x$ ) and adding the resulting equations we obtain (4.17), that is, $\partial f$ is semi-submonotone at $x_{0}$.

$(i i) \Longrightarrow(i v)$. Let us assume that $\partial f$ is semi-submonotone on $U$. Then Spingarn established (see [31, Proposition 2.5]) that $f$ is d-regular at every point of $U$, for the case $X=\mathbb{R}^{n}$. However the same proof works for an arbitrary real Banach space $X$.

To prove $M$-subsmoothness, fix $x_{0}$ in $U$ and take any sequence $\left\{x_{n}\right\}_{n} \subset X$, any $e \in S_{X}$ and any $\left\{x_{n}^{*}\right\}_{n} \subset X^{*}$ such that $x_{n}^{*} \in \partial f\left(x_{n}\right), \lim _{n \rightarrow+\infty} x_{n}=x_{0}$ and

$$
\lim _{n \rightarrow+\infty}\left\|\frac{x_{n}-x_{0}}{\left\|x_{n}-x_{0}\right\|}-e\right\|=0 .
$$

We may suppose that $x_{n} \neq x_{0}$, for all $n \in \mathbb{N}$. We note that (4.17) yields

$$
\liminf _{n \rightarrow+\infty} \inf _{z_{n}^{*} \in \partial f\left(x_{n}\right)}\left\langle z_{n}^{*}, \frac{x_{n}-x_{0}}{\left\|x_{n}-x_{0}\right\|}\right\rangle \geq \limsup _{n \rightarrow+\infty} \sup _{x_{0}^{*} \in \partial f\left(x_{0}\right)}\left\langle x_{0}^{*}, \frac{x_{n}-x_{0}}{\left\|x_{n}-x_{0}\right\|}\right\rangle .
$$

Using the continuity of the function $u \rightarrow f^{0}\left(x_{0} ; u\right)$ (see [4, Proposition 2.1.1]), we infer in particular that

$$
\liminf _{n \rightarrow+\infty}\left\langle x_{n}^{*}, e\right\rangle \geq f^{0}\left(x_{0} ; e\right)=\sup _{x_{0}^{*} \in \partial f\left(x_{0}\right)}\left\langle x_{0}^{*}, e\right\rangle .
$$

Since the operator $\partial f$ is $\left(\|\cdot\|, w^{*}\right)$-upper continuous at $x_{0}$ (see [4, Proposition 2.1.5]), we get

$$
\limsup _{n \rightarrow+\infty}\left\langle x_{n}^{*}, e\right\rangle \leq f^{0}\left(x_{0} ; e\right) .
$$

From (4.21), (4.22) and the directional regularity of $f$ at $x_{0}$ we conclude that $\left\langle x_{n}^{*}, e\right\rangle \rightarrow f^{\prime}\left(x_{0} ; e\right)$, that is, $f$ is $M$-semismooth at $x_{0}$.

Let us now suppose that $X=\mathbb{R}^{n}$. In this case, the implication $(i v) \Longrightarrow(i i)$ is [31, Proposition 2.4]. It remains to establish $(i i) \Longrightarrow(i)$. For this, let us suppose that $\partial f$ is semi-submonotone on $U$. Then $f$ is d-regular on $U$ (see the proof of $($ ii $) \Longrightarrow($ iv $)$ above). We only have to show that for every $x_{0} \in U$, relation (4.18) holds true. To this end, fix $\varepsilon>0$ and take $\delta>0$ such that relation (4.17) holds 
for $\varepsilon / 2$. Since $\partial f$ is upper semicontinuous at $x_{0}$, with no loss of generality we may suppose that

$$
\bigcup_{z \in B\left(x_{0}, \delta\right)} \partial f(z) \subset \partial f\left(x_{0}\right)+B\left(0, \frac{\varepsilon}{2}\right) .
$$

Let us now fix any $x \in B\left(x_{0}, \delta\right)$ and any $\left.t \in\right] 0,1\left[\right.$ and let us set $x_{t}=t x+(1-t) x_{0}$. Then applying Lebourg's Mean Value theorem ([17, Theorem 1.7]) on the segment $\left[x, x_{t}\right]$ we get $z_{1} \in\left[x, x_{t}\left[\right.\right.$ and $z_{1}^{*} \in \partial f\left(z_{1}\right)$ such that

$$
\left\langle z_{1}^{*}, x_{t}-x\right\rangle=f\left(x_{t}\right)-f(x) .
$$

Similarly, there exists $z_{2} \in\left[x_{0}, x_{t}\left[\right.\right.$ and $z_{2}^{*} \in \partial f\left(z_{2}\right)$

$$
\left\langle z_{2}^{*}, x_{t}-x_{0}\right\rangle=f\left(x_{t}\right)-f\left(x_{0}\right) .
$$

Since $x_{t}-x=(1-t)\left(x_{0}-x\right)$ and $x_{t}-x_{0}=t\left(x-x_{0}\right)$, multiplying (4.24) by $t$ and (4.25) by $(1-t)$, and adding the resulting equalities we get

$$
t f(x)+(1-t) f\left(x_{0}\right)-f\left(x_{t}\right)=t(1-t)\left\langle z_{1}^{*}-z_{2}^{*}, x-x_{0}\right\rangle .
$$

From (4.23) we may choose $x_{0}^{*} \in \partial f\left(x_{0}\right)$ such that

$$
\left\|z_{2}^{*}-x_{0}^{*}\right\|<\varepsilon / 2 .
$$

Applying (4.17) we get

$$
\left\langle z_{1}^{*}-x_{0}^{*}, \frac{z_{1}-x_{0}}{\left\|z_{1}-x_{0}\right\|}\right\rangle \geq-\frac{\varepsilon}{2} .
$$

Using (4.27) and the fact that

$$
\frac{z_{1}-x_{0}}{\left\|z_{1}-x_{0}\right\|}=\frac{x-x_{0}}{\left\|x-x_{0}\right\|},
$$

we deduce from (4.28) that

$$
\left\langle z_{1}^{*}-z_{2}^{*}, \frac{x-x_{0}}{\left\|x-x_{0}\right\|}\right\rangle \geq-\varepsilon .
$$

Combining the above formula with (4.26) we obtain (4.18), that is, $f$ is semismooth at $x_{0}$.

4.4. Subsmooth epi-Lipschitz sets. This last section is devoted to the study of subsmooth (or semi-subsmooth) epi-Lipschitz sets as epigraphical characterizations of the aforementioned classes of functions. The derived results are summarized in a diagram at the end of this section.

We recall ([26, 27]) the following definition.

Definition 4.10. A closed set $C$ is called epi-Lipschitz at $u_{0} \in C$ with respect to the direction $d \in X$ if there exists $\varepsilon>0, \delta>0$ such that for all $d^{\prime} \in B(d, \varepsilon)$, all $u \in C \cap B\left(u_{0}, \varepsilon\right)$ and all $\left.t \in\right] 0, \delta\left[\right.$ we have $u+t d^{\prime} \in C$.

Remark 4.11. (i) Every set $C$ is epi-Lipschitz at every $u_{0} \in$ int $C$ (with respect to any $d \in X)$.

(ii) If $u_{0} \in b d C$ (the boundary of $C$ ), then $C$ is epi-Lipschitz at $u_{0}$ with respect to $d \neq 0$ if, and only if, the set $C$ can be represented in a neighbourhood of $u_{0}$ as the epigraph of a Lipschitz continuous function $f$ (see [26], [27]), which is called a locally Lipschitz representation of $C$ at $u_{0}$. This means that there exists a 
topological complement $X_{d}$ of $\mathbb{R} d:=\{t d: t \in \mathbb{R}\}$ in $X$ (that is, $X=X_{d} \oplus \mathbb{R} d$ ), a neighbourhood $U$ of $u_{0}$ and a locally Lipschitz function $f: X_{d} \rightarrow \mathbb{R}$ such that

$$
C \cap U=\left\{x \oplus s d: x \in X_{d}, f(x) \leq s\right\} \cap U .
$$

(Here $X_{d}$ is endowed with the norm induced by the norm of $X$.) We denote by $\pi$ : $X \rightarrow X_{d}$ and $\rho: X \rightarrow \mathbb{R}$ the continuous linear mappings satisfying $u=\pi(u) \oplus \rho(u) d$ for all $u \in X$, and by $A: X_{d} \times \mathbb{R} \rightarrow X$ the continuous linear mapping given by $A(x, s)=x \oplus s d$, so that $\Lambda:=A^{-1}: X \rightarrow X_{d} \times \mathbb{R}$ is given by $\Lambda(u)=(\pi(u), \rho(u))$. We endow $X_{d} \times \mathbb{R}$ with the product norm $(x, s) \rightarrow\left(\|x\|^{2}+s^{2}\right)^{1 / 2}$. Consequently, (4.29) is equivalent to

$$
C \cap U=A(\text { epi } f) \cap U .
$$

We also define $F: X_{d} \rightarrow X$ by $F(x)=x \oplus f(x) d$, so that $F(x)=A((x, f(x))$. Consequently, if $k>0$ is the Lipschitz constant of $f$ in a neighbourhood $V$ of $x_{0}:=\pi\left(u_{0}\right)$ with $F(V) \subset U$, then it follows easily that

$$
\left\|F\left(x_{1}\right)-F\left(x_{2}\right)\right\| \leq \alpha\left\|x_{1}-x_{2}\right\|, \text { for all } x_{1}, x_{2} \in V,
$$

where

$$
\alpha=\|A\|\left(k^{2}+1\right)^{1 / 2} .
$$

In the sequel, we shall need the following lemma.

Lemma 4.12. Let $A: Z_{1} \rightarrow Z_{2}$ be an isomorphism between the Banach spaces $Z_{1}$ and $Z_{2}$, let $C$ be a non-empty closed subset of $Z_{1}$ and let $u_{0} \in C$. Then

(a) $A\left(T\left(C ; u_{0}\right)\right)=T\left(A(C) ; A\left(u_{0}\right)\right)$;

(b) $N\left(C ; u_{0}\right)=A^{*}\left(N\left(A(C) ; A\left(u_{0}\right)\right)\right)$.

Proof. Assertion (a) follows easily from the definition of Clarke's tangent cone (see formula (2.2) ) and the fact that $A$ is an isomorphism. We infer from $(a)$ that $\left(A\left(T\left(C ; u_{0}\right)\right)\right)^{o}=N\left(A(C) ;\left(A\left(u_{0}\right)\right)\right)$. Now the desired equality $(b)$ is a direct consequence of the fact that the adjoint operator $A^{*}$ is an isomorphism between the dual spaces $Z_{2}^{*}$ and $Z_{1}^{*}$.

In finite dimensions, Clarke, Stern and Wolenski have shown that proximal smoothness characterizes the epigraphs of lower $C^{2}$ functions (5, Section 5]). In an analogous way, an epi-Lipschitz set is prox-regular at $u$ if, and only if, the locally Lipschitz function $f$ given in (4.29) is lower- $C^{2}$ (see 25]). The forthcoming results will be built further in this line of research. Let us first establish the following lemma. In several statements below, $\pi$ will refer to the notation of Remark 4.11( $(i i)$.

Lemma 4.13. Let $C$ be an epi-Lipschitz set, let $u_{0} \in b d C$ and suppose that $N^{r}(C ;$. is hypomonotone (respectively, submonotone, semi-submonotone) at $u_{0}$ for some $r>0$.

Then, for every locally Lipschitz representation $f$ of $C$ around $u_{0}$, the Clarke subdifferential $\partial f$ is hypomonotone (respectively, submonotone, semi-submonotone) at $\pi\left(u_{0}\right)$.

Proof. In the sequel we shall refer to the notation of Remark4.11( $i i)$. In particular, we suppose that (4.29) holds and that $f$ has a Lipschitz constant $k>0$ on an open neighbourhood $V$ of $x_{0}=\pi\left(u_{0}\right)$ and that $F(V) \subset U$.

Let us prove the statement for submonotonicity. With no loss of generality we may suppose that $N^{r}(C ;$.$) is submonotone at u_{0}$, where

$$
r=\left\|\Lambda^{*}\right\|\left(k^{2}+1\right)^{1 / 2} .
$$


( $\Lambda^{*}$ denotes the adjoint of $\Lambda=A^{-1}$.) In order to establish that $\partial f$ is submonotone at $\pi\left(u_{0}\right)=x_{0}$, let $\varepsilon>0$ and set $\varepsilon_{1}=\alpha^{-1} \varepsilon$, where $\alpha$ is given by (4.31). Thus there exists $\delta>0$ with $B\left(u_{0}, \delta\right) \subset U$ such that for all $u_{1}, u_{2} \in B\left(u_{0}, \delta\right)$ and all $u_{i}^{*} \in N^{r}\left(C ; u_{i}\right), i \in\{1,2\}$,

$$
\left\langle u_{1}^{*}-u_{2}^{*}, u_{1}-u_{2}\right\rangle \geq-\varepsilon_{1}\left\|u_{1}-u_{2}\right\| .
$$

Let $\delta_{1} \leq \alpha^{-1} \delta$ such that $B\left(x_{0}, \delta_{1}\right) \subset V$. Then (4.30) implies that $F\left(B\left(x_{0}, \delta_{1}\right)\right) \subset$ $B\left(u_{0}, \delta\right)$. Consider now any two points $x_{1}, x_{2} \in B\left(x_{0}, \delta_{1}\right)$ and let $x_{i}^{*} \in \partial f\left(x_{i}\right)$, for $i \in\{1,2\}$. Then $\left(x_{i}^{*},-1\right) \in N\left(\right.$ epi $\left.f ;\left(x_{i}, f\left(x_{i}\right)\right)\right)$. Since $\Lambda$ is an isomorphism between the spaces $X$ and $X_{d} \times \mathbb{R}$ and since $C \cap U=A($ epi $f) \cap U$, applying Lemma 4.12 for the points

$$
u_{i}:=F\left(x_{i}\right)=A\left(x_{i}, f\left(x_{i}\right)\right), \quad i \in\{1,2\},
$$

we get $u_{i}^{*}:=\Lambda^{*}\left(\left(x_{i}^{*},-1\right)\right) \in N\left(C ; u_{i}\right), i \in\{1,2\}$. Since $\left\|x_{i}^{*}\right\| \leq k$, for $i \in\{1,2\}$ (see [4, Proposition 2.1.2 (a)] e.g.), we obtain

$$
\left\|u_{i}^{*}\right\| \leq\left\|\Lambda^{*}\right\|\left(\left\|x_{i}^{*}\right\|^{2}+1\right)^{1 / 2} \leq r, \quad \text { so that } u_{i}^{*} \in N^{r}\left(C ; u_{i}\right) .
$$

From the above and (4.32) we infer that

$$
\left\langle\Lambda^{*}\left(x_{1}^{*},-1\right)-\Lambda^{*}\left(x_{2}^{*},-1\right), F\left(x_{1}\right)-F\left(x_{2}\right)\right\rangle \geq-\varepsilon \alpha^{-1}\left\|F\left(x_{1}\right)-F\left(x_{2}\right)\right\|,
$$

or equivalently, in view of (4.30) and (4.33)

$$
\left\langle x_{1}^{*}-x_{2}^{*}, x_{1}-x_{2}\right\rangle \geq-\varepsilon\left\|x_{1}-x_{2}\right\| .
$$

Consequently, $\partial f$ is submonotone at $x_{0}$.

To establish the corresponding statement for semi-submonotonicity, it suffices to replace $x_{2}$ by $x_{0}$ and $x_{2}^{*} \in \partial f\left(x_{2}\right)$ by $x_{0}^{*} \in \partial f\left(x_{0}\right)$ in the above proof. Finally, assuming that $N^{r}(C ;$.$) is hypomonotone at u_{0}$ relative to the parameters $\sigma>0$ and $\delta>0$ (see (4.1) ), one again considers $\delta_{1} \leq \alpha^{-1} \delta$ such that $B\left(x_{0}, \delta_{1}\right) \subset V$ and $\sigma_{1}=\alpha^{-2} \sigma$. Making obvious modifications in the above proof one establishes analogously the hypomonotonicity of $\partial f$ at $x_{0}$.

We are now ready to establish the results that motivated the study of the paper. The first one relates the class of subsmooth sets (introduced in Definition 3.1) to the approximately convex functions.

Theorem 4.14. Let $X$ be a Banach space, let $C$ be an epi-Lipschitz subset of $X$, and let $u_{0} \in b d C$. Then the following statements are equivalent:

(i) $C$ is subsmooth at $u_{0}$;

(ii) every locally Lipschitz representation $f$ of $C$ at $u_{0}$ is approximately convex at $\pi\left(u_{0}\right)$

(iii) some locally Lipschitz representation $f$ of $C$ at $u_{0}$ is approximately convex at $\pi\left(u_{0}\right)$.

Proof. Implication $(i i) \Longrightarrow($ iii $)$ is obvious and implication $(i) \Longrightarrow(i i)$ follows from Lemma 4.13 and Theorem $4.5(i i) \Longrightarrow(i)$. Thus, it remains to show $(i i i) \Longrightarrow(i)$. To this end, referring to the notation of Remark 4.11 ii), let us suppose that $f$ is approximately convex at $x_{0}=\pi\left(u_{0}\right)$, and locally Lipschitz of constant $k>0$ on $V$ with $F(V) \subset U$ (see (4.30)). In view of Remark 3.3( $(i)$ it clearly suffices to show that $N^{1}(C ;$.$) is submonotone at u_{0}$. Fix any $\varepsilon>0$ and put

$$
\varepsilon_{1}=\frac{\varepsilon}{2\|A\| \cdot\|\Lambda\|} .
$$


According to implication $(i) \Longrightarrow\left(\right.$ iii) of Theorem 4.5 , there exists $\delta_{1}>0$ (with $\left.B\left(x_{0}, \delta_{1}\right) \subset V\right)$ such that by (4.14) one has

$$
f\left(x_{2}\right) \geq f\left(x_{1}\right)+\left\langle x_{1}^{*}, x_{2}-x_{1}\right\rangle-\varepsilon_{1}\left\|x_{1}-x_{2}\right\|,
$$

for all $x_{1}, x_{2} \in B\left(x_{0}, \delta_{1}\right)$ and all $x_{1}^{*} \in \partial f\left(x_{1}\right)$. Take $\delta \leq \delta_{1} /\|\Lambda\|$ so that $B\left(u_{0}, \delta\right) \subset$ $U$. Consider any $u_{i} \in C \cap B\left(u_{0}, \delta\right)$ and $u_{i}^{*} \in N^{1}\left(C ; u_{i}\right), i \in\{1,2\}$. We claim that

$$
\left\langle u_{1}^{*}, u_{2}-u_{1}\right\rangle \leq \frac{\varepsilon}{2}\left\|u_{1}-u_{2}\right\| .
$$

To this end, let $x_{i}=\pi\left(u_{i}\right)$ and $t_{i}=\rho\left(u_{i}\right)$, so that $u_{i}=A\left(x_{i}, t_{i}\right)$ and $t_{i} \geq f\left(x_{i}\right)$, $i \in\{1,2\}$. If $t_{1}>f\left(x_{1}\right)$, then $u_{1} \in \operatorname{int} C$ and consequently $N\left(C ; u_{1}\right)=\{0\}$, so (4.35) holds trivially. Thus, we may suppose that $t_{1}=f\left(x_{1}\right)$ and $u_{1}^{*} \neq 0$. In view of Lemma 4.12 $u_{1}^{*} \in(\Lambda)^{*}\left(N(\operatorname{epi} f) ;\left(x_{1}, f\left(x_{1}\right)\right)\right)$. Since $\left\|u_{1}^{*}\right\| \leq 1$, there exist $0<\lambda \leq\|A\|$ and $x_{1}^{*} \in \partial f\left(x_{1}\right)$ such that

$$
u_{1}^{*}=\lambda(\Lambda)^{*}\left(x_{1}^{*},-1\right) .
$$

Moreover, taking the choice of $\delta$ into account, it is easily seen that $x_{1}, x_{2} \in B\left(x_{0}, \delta_{1}\right)$ and that

$$
\left\|x_{1}-x_{2}\right\| \leq\|\Lambda\| \cdot\left\|u_{1}-u_{2}\right\| .
$$

Since $t_{2} \geq f\left(x_{2}\right)$, we obtain from (4.34) and (4.37) that

$$
\left\langle\left(x_{1}^{*},-1\right),\left(x_{2}, t_{2}\right)-\left(x_{1}, f\left(x_{1}\right)\right)\right\rangle \leq \varepsilon_{1}\left\|x_{1}-x_{2}\right\| \leq \varepsilon_{1}\|\Lambda\| \cdot\left\|u_{1}-u_{2}\right\| .
$$

Thus

$$
\left\langle\lambda\left(x_{1}^{*},-1\right), \Lambda\left(u_{2}\right)-\Lambda\left(u_{1}\right)\right\rangle \leq \varepsilon_{1}\|A\| .\|\Lambda\| .\left\|u_{1}-u_{2}\right\|=\frac{\varepsilon}{2}\left\|u_{1}-u_{2}\right\|,
$$

which, in view of (4.36) yields (4.35). Now interchanging the roles of $u_{1}$ and $u_{2}$ in (4.35) we obtain

$$
\left\langle u_{2}^{*}, u_{1}-u_{2}\right\rangle \leq \frac{\varepsilon}{2}\left\|u_{1}-u_{2}\right\| .
$$

Adding the inequalities (4.35) and (4.38) we obtain (3.2).

The next theorem concerns the class of semi-subsmooth sets. Comparing with the epigraphical characterization of Theorem 4.14 there are two important differences:

- the statements are now local (and not pointwise);

- the equivalence is established only in finite dimensions.

Theorem 4.15. Let $C$ be an epi-Lipschitz subset of $\mathbb{R}^{n}$ and $u_{0} \in b d C$. Then the following statements are equivalent:

(i) $C$ is semi-subsmooth around $u_{0}$;

(ii) every locally Lipschitz representation $f$ of $C$ at $u_{0}$ is semismooth and $d$ regular around $\pi\left(u_{0}\right)$;

(iii) some locally Lipschitz representation $f$ of $C$ at $u_{0}$ is semismooth and $d$ regular around $\pi\left(u_{0}\right)$.

Proof. Implication $(i i) \Longrightarrow($ iii $)$ is obvious. Let us prove $(i) \Longrightarrow(i i)$. If $f$ is a locally Lipschitz representation of $C$ in a neighbourhood of $u_{0}$, applying Lemma 4.13 we obtain that $\partial f$ is semi-submonotone in a neighbourhood of $\pi\left(u_{0}\right)$. From $(i i) \Longrightarrow(i)$ of Theorem 4.9 we obtain $(i i)$. Finally, implication $(i i i) \Longrightarrow(i)$ follows in the same way as in Theorem 4.14 One uses (4.19) in place of (4.14) to obtain (3.3). The proof is complete. 
The following theorem completes the trilogy of epi-graphical characterizations. It concerns the class of prox-regular sets (see [33] or [5] for the finite-dimensional case).

Theorem 4.16. Let $X$ be a Hilbert space, let $C$ be an epi-Lipschitz subset of $X$, and let $u_{0} \in b d C$. Then the following statements are equivalent:

(i) $C$ is prox-regular at $u_{0}$;

(ii) every locally Lipschitz representation $f$ of $C$ at $u_{0}$ is weakly convex at $\pi\left(u_{0}\right)$;

(iii) some locally Lipschitz representation $f$ of $C$ at $u_{0}$ is weakly convex at $\pi\left(u_{0}\right)$.

Proof. The proof follows the lines of the proof of Theorem 4.14 above.

As before, Lemma 4.13 and Theorem $4.1(i i) \Longrightarrow(i)$ entail implication $(i) \Longrightarrow(i i)$, while implication $(i i) \Longrightarrow(i i i)$ is obvious. Let us establish $(i i i) \Longrightarrow(i)$. Using the same notation as before, it is enough to establish that $N^{1}(C ;$.$) is hypomonotone$ at $u_{0}$. By Theorem 4.1 (iii) there exist $\sigma_{1}>0$ and $\delta_{1}>0$ (with $B\left(x_{0}, \delta_{1}\right) \subset V$ ) such that (4.4) holds for $\sigma_{1}$. Take $\delta \leq \delta_{1} /\|\Lambda\|$ such that $B\left(u_{0}, \delta\right) \subset U$ and put $\sigma=2 \sigma_{1}\|A\| \cdot\|\Lambda\|^{2}$. Then one shows in an analogous way as in Theorem 4.14 that for all $u_{i} \in C \cap B\left(u_{0}, \delta\right)$ and $u_{i}^{*} \in N^{1}\left(C ; u_{i}\right), i \in\{1,2\}$,

$$
\max \left\{\left\langle u_{1}^{*}, u_{2}-u_{1}\right\rangle,\left\langle u_{2}^{*}, u_{1}-u_{2}\right\rangle\right\} \leq \frac{\sigma}{2}\left\|u_{1}-u_{2}\right\|^{2} .
$$

This yields (3.1) and finishes the proof.

Finally, putting together the results of this section we obtain the following corollary.

Corollary 4.17. Let $f$ be a locally Lipschitz function on a Banach space $X$. Then

epi $f$ is subsmooth $\Leftrightarrow f$ is approximately convex $\Leftrightarrow \partial f$ is submonotone.

If $X$ is a Hilbert space, then

epi $f$ is prox-regular $\Leftrightarrow f$ is weakly convex $\Leftrightarrow \partial f$ is hypomonotone.

If $X=\mathbb{R}^{n}$, then

$$
\begin{aligned}
\text { epi } f \text { is semi-subsmooth } & \Leftrightarrow f \text { is d-regular and semi-smooth } \\
& \Leftrightarrow \partial f \text { is semi-submonotone. }
\end{aligned}
$$

Let us finally notice that if a set $C$ is prox-regular at $u_{0}$, then for some $\delta>0$ and $r>0$ and for every $u, v \in C \cap B\left(u_{0}, \delta\right)$ and $u^{*} \in N^{1}(C ; u)$,

$$
\left\langle u^{*}, v-u\right\rangle \leq \frac{r}{2}\|v-u\|^{2} \text {. }
$$

We call this property the local "paint-roller property", following [5, 6] e.g., where the corresponding global property is considered. In some sense it reflects the degree of smoothness of the boundary of $C$, since it asserts that the set $C$ can be "supported" from the exterior by a parabola of a fixed ratio at all points near $u_{0}$. An analogous statement can be derived for epi-Lipschitz subsmooth sets as a consequence of Theorem 4.14 .

Corollary 4.18. An epi-Lipschitz set $C$ is subsmooth at $u_{0}$ if, and only if, there exists $d \in X, \delta>0$ and a non-negative function $\varphi \in C_{*}^{1}\left(\mathbb{R}, \mathbb{R}_{+}\right)$such that for every $u, v \in C \cap B\left(u_{0}, \delta\right)$ and $u^{*} \in N^{1}(C ; u)$

$$
\left\langle u^{*}, v-u\right\rangle \leq \varphi(\|\pi(v)-\pi(u)\|),
$$


where $\pi$ denotes the projection associated with one of the locally Lipschitz representations of $C$ at $u_{0}$, according to the notation of Remark 4.11.

Proof. [ $\Longrightarrow$ ] Suppose that the epi-Lipschitz set $C$ is subsmooth at $u_{0}$. If $u_{0} \in \operatorname{int} C$, then $N^{1}\left(C ; u_{0}\right)=\{0\}$ and (4.41) holds trivially for $\varphi \equiv 0$. So we may suppose that $u_{0} \in$ bd $C$. Then by Remark 4.11 there exists some $d \in X$ such that $X=X_{d} \oplus \mathbb{R} d$, some $\delta>0$, and some locally Lipschitz function $f$ on $X_{d}$, such that

$$
C \cap B\left(u_{0}, \delta\right)=\left\{x \oplus s d: x \in X_{d}, f(x) \leq s\right\} \cap B\left(u_{0}, \delta\right) .
$$

According to the notation of Remark4.11we denote by $\pi: X \rightarrow X_{d}$ and $\rho: X \rightarrow \mathbb{R}$ the continuous linear mappings satisfying $u=\pi(u) \oplus \rho(u) d$, for all $u \in X$, and by $A: X_{d} \oplus \mathbb{R} \rightarrow X$ the isomorphism $A(x, s)=x \oplus s d$, so that $A^{-1}: X \rightarrow X_{d} \oplus \mathbb{R}$ is given by $A^{-1}(u)=(\pi(u), \rho(u))$. Theorem 4.14 ensures that $f$ is approximately convex at $\pi\left(u_{0}\right)$. So, applying Theorem 4.5 (iv), we infer the existence of $\delta_{1}>0$ and a non-negative function $a \in C_{*}^{1}\left(\mathbb{R}, \mathbb{R}_{+}\right)$such that for all $x, y \in B\left(\pi\left(u_{0}\right), \delta_{1}\right)$, $x^{*} \in \partial f(x)$ and $\beta \geq f(y)$,

$$
\beta \geq f(x)+\left\langle x^{*}, y-x\right\rangle-a(\|y-x\|),
$$

or equivalently

$$
\left\langle\left(x^{*},-1\right),(y, \beta)-(x, f(x))\right\rangle \leq a(\|y-x\|) .
$$

Now let $\delta>0$ be such that $\pi\left(B\left(u_{0}, \delta\right)\right) \subset B\left(\pi\left(u_{0}\right), \delta_{1}\right)$, and let us show that (4.41) holds for $\varphi \equiv\|A\| a$. To this end, consider any $u, v \in C \cap B\left(u_{0}, \delta\right)$ and $u^{*} \in N^{1}(C ; u)$. We set $x=\pi(u), y=\pi(v)$. If $\rho(u)>f(x)$, then $u \in \operatorname{int} C$ and $N^{1}(C ; u)=\{0\}$, consequently (4.41) holds trivially since $\varphi \geq 0$. The same argument holds in the case $u^{*}=0$. Hence we may suppose that $\rho(u)=f(x)$ and $u^{*} \neq 0$. Since $A$ is an isomorphism and since $f$ is locally Lipschitz, using Lemma 4.12(b) we infer that $A^{*}\left(u^{*}\right)=t\left(x^{*},-1\right)$, for some $t>0$. This equality entails that $t\|A\|^{-1}\left(\left\|x^{*}\right\|^{2}+1\right)^{\frac{1}{2}} \leq 1$ and hence $t \leq\|A\|$. Then (4.43) yields for $\beta=\rho(v) \geq f(y)$

$$
t^{-1}\left\langle A^{*}\left(u^{*}\right),(y, \rho(v))-(x, f(x))\right\rangle \leq a(\|\pi(v)-\pi(u)\|) .
$$

Since $0<t \leq\|A\|$ we get

$$
\left\langle u^{*}, A(\pi(v), \rho(v))-A(\pi(u), \rho(u))\right\rangle \leq \varphi(\|\pi(v)-\pi(u)\|),
$$

or equivalently (4.41).

$[\Longleftarrow]$ We now assume that there exists $d \in X, \delta>0$ and $\varphi \in C_{*}^{1}\left(\mathbb{R}, \mathbb{R}_{+}\right)$such that (4.41) holds. Let us show that $C$ is subsmooth at $u_{0}$. To this end, let $\varepsilon>0$. Since $\varphi^{\prime}(0)=\varphi(0)=0$, there exists $0<\delta_{1}<\delta$ such that for all $0<t<\delta_{1}$,

$$
\frac{\varphi(t)}{t} \leq \frac{\varepsilon}{2\|\pi\|}
$$

Then for all $u_{1}, u_{2} \in B\left(u_{0}, \delta_{1}\right), u_{i}^{*} \in N^{1}\left(C ; u_{i}\right), i \in\{1,2\}$, we have

$$
\left\langle u_{1}^{*}-u_{2}^{*}, u_{1}-u_{2}\right\rangle \geq-2 \varphi\left(\left\|\pi\left(u_{1}\right)-\pi\left(u_{2}\right)\right\|\right) .
$$

It follows from (4.44) and (4.45) that

$$
\left\langle u_{1}^{*}-u_{2}^{*}, u_{1}-u_{2}\right\rangle \geq-\varepsilon\left\|u_{1}-u_{2}\right\|,
$$

that is $C$ is subsmooth at $u_{0}$. 
The following diagram summarizes the situation. In this diagram $f: U \rightarrow \mathbb{R}$ is locally Lipschitz on the open subset $U$ of $\mathbb{R}^{n}, \partial f: U \rightrightarrows \mathbb{R}^{n}$ denotes its Clarke subdifferential and $C=$ epi $f$ stands for its epigraph.

\begin{tabular}{|c|c|c|c|c|}
\hline $\begin{array}{c}T=\partial f \\
\text { (locally bounded) }\end{array}$ & & $\begin{array}{c}f: U \rightarrow \mathbb{R} \\
\text { (locally Lipschitz) }\end{array}$ & & $\begin{array}{c}C=\operatorname{epi} f \\
\text { (epi-Lipschitz) }\end{array}$ \\
\hline $\begin{array}{c}\text { monotone } \\
\Downarrow\end{array}$ & $\Longleftrightarrow$ & $\begin{array}{c}\text { convex function } \\
\Downarrow\end{array}$ & $\Longleftrightarrow$ & $\begin{array}{c}\text { convex set } \\
\Downarrow\end{array}$ \\
\hline $\begin{array}{c}\text { hypomonotone } \\
\Downarrow\end{array}$ & $X \stackrel{\text { Hilbert }}{\Longleftrightarrow}$ & $\begin{array}{c}\text { weakly convex } \\
\Downarrow\end{array}$ & $X \stackrel{\text { Hilbert }}{\Longleftrightarrow}$ & $\begin{array}{c}\text { prox-regular } \\
\Downarrow\end{array}$ \\
\hline $\begin{array}{c}\text { submonotone } \\
\Downarrow\end{array}$ & $\Longleftrightarrow$ & $\begin{array}{c}\text { approximately convex } \\
\Downarrow\end{array}$ & $\Longleftrightarrow$ & $\begin{array}{c}\text { subsmooth } \\
\Downarrow\end{array}$ \\
\hline semi-submonotone & $\stackrel{X=\mathbb{R}^{n}}{\Longleftrightarrow}$ & $\begin{array}{l}\text { semismooth } \\
\& \text { d-regular }\end{array}$ & $\stackrel{X=\mathbb{R}^{n}}{\Longleftrightarrow}$ & semi-subsmooth \\
\hline
\end{tabular}

\section{REFERENCES}

1. Aussel, D., Corvellec, J.-N. \& Lassonde, M., Subdifferential characterization of quasiconvexity and convexity, J. Convex Anal. 1 (1994), 195-201. MR.1363111 (97e:49012)

2. Bernard, F. \& Thibault, L., Uniform Prox-regularity of functions and epigraphs in Hilbert spaces, Nonlinear Anal. TMA, to appear.

3. Bounkhel, M. \& Thibault, L., On various notions of regularity of sets in non-smooth analysis, Nonlinear Anal. TMA 48 (2002), 223-246. MR1870754 (2002j:46048)

4. Clarke, F., Optimization and non-smooth analysis, Wiley Interscience, New York 1983 (Republished in 1990: Vol. 5, Classics in Applied Mathematics, Society for Industrial and Applied Mathematics, Philadelphia, Pa.). MR1058436 (91e:49001)

5. Clarke, F., Stern, R. \& Wolenski, P., Proximal smoothness and the lower-C ${ }^{2}$ property, J. Convex Anal. 2 (1995), 117-144. MR1363364 (96j:49014)

6. Colombo, G. \& Goncharov, V., Variational inequalities and regularity properties of closed sets in Hilbert spaces, J. Convex Anal. 8 (2001), 197-221. MR.1829062 (2002f:49031)

7. Correa, R. \& Jofré, A., Tangentially continuous directional derivatives in nonsmooth analysis, J. Optim. Theory Appl. 61 (1989), 1-21. MR0993912 (90h:49009)

8. Correa, R., Jofré, A. \& Thibault, L., Subdifferential monotonicity as characterization of convex functions, Num. Func. Anal. Optim. 15 (1994), 531-535. MR1281560 (95d:49029)

9. Danillidis, A. \& Georgiev, P., Approximate convexity and submonotonicity, J. Math. Anal. Appl. 291 (2004), 292-301. MR2034075|(2004m:49048)

10. Danillidis, A., Georgiev, P. \& Penot, J.-P., Integration of multivalued operators and cyclic submonotonicity, Trans. Amer. Math. Soc. 355 (2003), 177-195. MR.1928084(2003h:49030)

11. Deville, R. Godefroy, G. \& Zizler, V., Smoothness and renormings in Banach spaces. Pitman Monographs and Surveys in Pure and Applied Mathematics, 64. Longman Scientific \& Technical, Harlow; co-published in the United States with John Wiley \& Sons, Inc., New York, 1993. MR1211634 (94d:46012)

12. Ekeland, I., Nonconvex minimization problems, Bull. Amer. Math. Soc. 1 (1979), 443-474. MR0526967 (80h:49007)

13. FABIÁN, M., Subdifferentiability and trustworthiness in the light of a new variational principle of Borwein and Preiss, Acta Univ. Carolin. Math. Phys. 30 (1989), 51-56. MR1046445 (91c:49024)

14. Federer, H., Curvature measures, Trans. Amer. Math. Soc. 93 (1959), 418-491. MR0110078 $(22: 961)$

15. Hiriart-UrRuty, J.-B., "Generalized differentiability, duality and optimization for problems dealing with differences of convex functions", in: Convexity and Duality in Optimization", Lecture Notes in Econom. Math. Systems 256 (1984), 37-70. MR0873269|(88b:90101)

16. Kruger, A.Y., $\varepsilon$-semidifferentials and $\varepsilon$-normal elements, Depon. VINITI, No. 1331-81, Moscow, 1981 (in Russian).

17. Lebourg, G., Generic differentiability of Lipschitzian functions, Trans. Amer. Math. Soc. 256 (1979), 125-144. MR 0546911 (80i:58012)

18. LEWIS, A., Robust regularization, preprint CECM, 17p, 2002. 
19. Marcellin, S., Initiation à l'analyse séquentielle non lisse dans les espaces d'Asplund, Master Thesis, 142p, (Montpellier, 2002).

20. Mifflin, R., Semismooth and semi-convex functions in constrained optimization, SIAM J. Control Optim. 15 (1977), 959-972. MR0461556 (57:1541)

21. Mordukhovich, B.S., ShaO, Y., Nonsmooth sequential analysis in Asplund spaces, Trans. Amer. Math. Soc. 348 (1996), 1235-1280. MR.1333396 (96h:49036)

22. NGai, H.V., ThÉrA, M., "On $\varepsilon$-convexity and $\varepsilon$-monotonicity", in: Calculus of Variations and Differential Equations, A. Ioffe, S. Reich, and I. Shafrir (Eds.), Research Notes in Mathematical Series (Chapman \& Hall, 82-100, 1999). MR1713840 (2000h:49027)

23. Ngai, H.V., Luc, D.T. \& Thera, M., Approximate convex functions, J. Nonlinear Convex Anal. 1 (2000), 155-176. MF 1777137(2001e:49032)

24. Penot, J.-P., Favorable classes of mappings and multimappings in nonlinear analysis and optimization, J. Convex Anal. 3 (1996), 97-116. MR.1422755 (97i:90110)

25. Poliquin, R., Rockafellar, R.T. \& Thibault, L., Local differentiability of distance functions, Trans. Amer. Math. Soc. 352 (2000), 5231-5249. MR.1694378(2001b:49024)

26. Rockafellar, R.T., Clarke's tangent cones and the boundaries of closed sets in $\mathbb{R}^{n}$, NonLinear Anal. TMA 3 (1979), 145-154. MR.0520481 (80d:49032)

27. Rockafellar, R.T., Generalized directional derivatives and subgradients of nonconvex functions, Canad. J. Math. 32 (1980), 257-280. MR0571922 (81f:49006)

28. Rockafellar, R.T., "Favorable Classes of Lipschitz Continuous Functions in Subgradient Optimization" in Nondifferentiable Optimization (1982), Nurminski E. (eds.), Pergamon Press, New York. MR0704977 (85e:90069)

29. Rolewicz, S., On the coincidence of some subdifferentials in the class of $\alpha($.$) -paraconvex$ functions, Optimization 50 (2001), 353-363. MR.1892909 (2003e:49033)

30. Shapiro, A., Existence and differentiability of metric projections in Hilbert spaces, SIAM J. Optimization 4 (1994), 130-141. MR1260410 (94m:90111)

31. Spingarn, J.E., Submonotone subdifferentials of Lipschitz functions, Trans. Amer. Math. Soc. 264 (1981), 77-89. MR0597868 (82g:26016)

32. Thibault, L., On the subdifferentials of optimal value functions, SIAM J. Control Optim. 29 (1991), 1019-1036. MR.1110085 (92e:49027)

33. Vial, J.-P., Strong and weak convexity of sets and functions, Math. Oper. Res. 8 (1983), 231-259. MR0707055 (84m:90107)

Département de Mathématiques, Université de Perpignan, 66860 Perpignan Cedex, FRANCE

E-mail address: aussel@univ-perp.fr

Departament de Matemàtiques, Universitat Autònoma de Barcelona, E-08193 Bellaterra (Cerdanyola del Vallès), Spain

E-mail address: arisd@mat.uab.es

Université Montpellier II, Département de Mathématiques, CC 051, Place Eugène Bataillon, 34095 Montpellier Cedex 05, France

E-mail address: thibault@math.univ-montp2.fr 